TRANSACTIONS OF THE

AMERICAN MATHEMATICAL SOCIETY

Volume 360, Number 9, September 2008, Pages 4639-4679

S 0002-9947(08)04413-9

Article electronically published on April 7, 2008

\title{
EXPONENTIAL SEPARATION AND PRINCIPAL FLOQUET BUNDLES FOR LINEAR PARABOLIC EQUATIONS ON GENERAL BOUNDED DOMAINS: NONDIVERGENCE CASE
}

\author{
JURAJ HÚSKA
}

\begin{abstract}
We consider the Dirichlet problem for linear nonautonomous second order parabolic equations of nondivergence type on general bounded domains with bounded measurable coefficients. Under such minimal regularity assumptions, we establish the existence of a principal Floquet bundle exponentially separated from a complementary invariant bundle. As a special case of our main theorem, assuming the coefficients are time-periodic, we obtain a new result on the existence of a principal eigenvalue of an associated (time-periodic) parabolic eigenvalue problem. We also show the existence of a uniform spectral gap between the principal eigenvalue and the rest of the spectrum for a class of time-periodic uniformly parabolic operators. Finally, we prove the uniqueness of positive entire solutions in the class of solutions whose supremum norms do not grow superexponentially as time goes to negative infinity.
\end{abstract}

\section{INTRODUCTION}

In this paper we consider linear nonautonomous nondivergence structure parabolic equations

$$
\begin{aligned}
u_{t}-L u=0 & \text { in } \quad \Omega \times J, \\
u=0 & \text { on } \quad \partial \Omega \times J .
\end{aligned}
$$

Here $\Omega \subset \mathbb{R}^{N}$ is an arbitrary bounded domain, $J$ is an open interval in $\mathbb{R}$, and $L$ is a time-dependent second-order elliptic operator of the nondivergence form

$$
L u=M u+c_{0} u=a_{i j}(x, t) D_{i j} u+b_{i}(x, t) D_{i} u+c_{0}(x, t) u
$$

(we use the summation convention and the notation $D_{i}=\partial / \partial x_{i}$ ). We assume that the coefficients of $L$ satisfy the following conditions.

Received by the editors April 26, 2006

2000 Mathematics Subject Classification. Primary 35K10; Secondary 35B05.

Key words and phrases. Exponential separation, nonsmooth domains, positive entire solutions, principal Floquet bundle, spectral gap.

The author was supported by the Doctoral Dissertation Fellowship of the University of Minnesota.

(C)2008 American Mathematical Society Reverts to public domain 28 years from publication 
(L1) $a_{i j} \in C(\Omega \times \mathbb{R}), b_{i}, c_{0}$ are measurable and for some positive constants $\alpha_{0}$, $\beta_{0}$ we have

$$
\begin{array}{r}
\left|a_{i j}(x, t)\right|,\left|b_{i}(x, t)\right|,\left|c_{0}(x, t)\right|<\beta_{0} \quad(i, j=1, \ldots, N, x \in \Omega, t \in \mathbb{R}), \\
a_{i j}(x, t) \xi_{i} \xi_{j} \geq \alpha_{0}|\xi|^{2} \quad\left(\xi \in \mathbb{R}^{N}, x \in \Omega, t \in \mathbb{R}\right) .
\end{array}
$$

In case $L$ is a time-independent (elliptic) or time-periodic operator, the existence of an associated principal eigenvalue and eigenfunction is well known and has been thoroughly studied (see, for example, [1, 3, 4, 7, 11]).

If $L$ is time-dependent with general time-dependence, one can still define objects analogous to principal eigenvalues and principal eigenfunctions, the relevant concepts being the principal spectrum, the principal Floquet bundle and the associated exponential separation. Investigation of the principal Floquet bundles and exponential separation has been carried out in $[13,14,15,16,17,22,24,25,26,27,31,33$. Typically, one proves the existence of two time-dependent spaces $X^{1}(t), X^{2}(t)$, $t \in \mathbb{R}$, which are subspaces of a suitable Banach space $X$ from which one takes the initial conditions; $X^{1}(t)$ is the (one-dimensional) span of a positive function, $X^{2}(t) \backslash\{0\}$ does not contain any nonnegative function and the subspaces are complementary to one another:

$$
X=X^{1}(t) \oplus X^{2}(t) \quad(t \in \mathbb{R}) .
$$

Moreover, $X^{1}(t)$ is uniquely determined by $\Omega$ and $L$ and the bundles $X^{i}(t), t \in \mathbb{R}$, $i=1,2$ are invariant for (1.1): if $u_{1}(t), u_{2}(t)$ are solutions with $u_{i}(s) \in X^{i}(s)$, then $u_{i}(t) \in X^{i}(t)$ for all $t>s$. Finally, if $u_{i}(s) \in X^{i}(s)(i=1,2)$, assuming $u_{1}$ is nontrivial, we have the estimate (which we call the exponential separation)

$$
\frac{\left\|u_{2}(\cdot, t)\right\|_{X}}{\left\|u_{1}(\cdot, t)\right\|_{X}} \leq C e^{-\gamma(t-s)} \frac{\left\|u_{2}(\cdot, s)\right\|_{X}}{\left\|u_{1}(\cdot, s)\right\|_{X}} \quad(t \geq s),
$$

where $C, \gamma>0$ are some positive constants. The one dimensional bundle $X^{1}(t)$, $t \in \mathbb{R}$, also known as the principal Floquet bundle, is an analogue of the principal eigenfunction and its uniqueness parallels the simplicity of the principal eigenvalue in the time-independent (or time-periodic) case. Moreover, exponential separation is a generalization of the fact that the principal eigenvalue of a time-independent elliptic (or time-periodic parabolic) operator is smaller than the real part of any other eigenvalue. We refer the reader to [5,6, 12-18, 22-27, 31-36] for the motivation and applications of the study of invariant bundles and exponential separation in nonautonomous parabolic equations with general time-dependence.

The motivation for our present work comes from the previous works on exponential separation cited above. In all of the references, where nondivergence equations are considered, some degree of smoothness of the domain is assumed to derive an estimate as in (1.4). The least restrictive of these references is [17, where the domain is assumed to be only Lipschitz and the regularity assumptions on the coefficients are the same as in (L1). In particular, at least for Lipschitz domains, the smoothness of the coefficients is irrelevant in establishing (1.3) and (1.4). It seems therefore natural to ask whether exponential separation can be derived without any smoothness assumptions on the domain. We have recently answered this question in the affirmative in [13] for divergence form equations. For nondivergence form equations on general domains such results are only available if $L$ in (1.2) has time-independent coefficients [3], a much simpler case than the one with general time-dependence considered here. 
It is our goal, inspired by the development in [3] and [13, to establish the existence of a principal Floquet bundle exponentially separated from a complementary invariant bundle for equations (1.1), whose coefficients satisfy (L1), on arbitrary bounded domains. Such an undertaking should also reveal what is the true underlying principle behind the results on exponential separation.

We find it instructive to first discuss some of the results proved in [17, where $\Omega$ is assumed to be Lipschitz. This will also enable us to show what difficulties we have to face in the general case. We denote by $u\left(\cdot, t ; s, u_{0}\right)(t \geq s)$ the solution of (1.1) with the initial condition $u(\cdot, s)=u_{0}$, where $u_{0} \in C_{0}(\bar{\Omega})$, the space of continuous functions vanishing on $\partial \Omega$. The following result is a special case of 17 , Theorem 2.2].

Theorem 1.1. Let $\Omega$ be a Lipschitz bounded domain. Let $u_{0} \in C_{0}(\bar{\Omega})$ be such that the solution $u\left(\cdot, t, s, u_{0}\right)$ vanishes somewhere in $\Omega$ for all $t>s$. Moreover, let $v$ be a positive solution of (1.1) on $\Omega \times(s-1, \infty)$. Then there are constants $C, \gamma>0$ such that

$$
\frac{\left\|u\left(\cdot, t ; s, u_{0}\right)\right\|_{L^{\infty}(\Omega)}}{\|v(\cdot, t)\|_{L^{\infty}(\Omega)}} \leq C e^{-\gamma(t-s)} \frac{\left\|u_{0}\right\|_{L^{\infty}(\Omega)}}{\|v(\cdot, s)\|_{L^{\infty}(\Omega)}} \quad(t \geq s) .
$$

Moreover, $C$ and $\gamma$ depend only on $N, \alpha_{0}$ and $\beta_{0}$ in (L1) and on the Lipschitz characteristics of $\Omega$.

Roughly speaking, Theorem 1.1 states that on Lipschitz domains sign-changing solutions are exponentially dominated by positive solutions. As a consequence of this theorem the authors of [17 have given the following characterization of the space $X^{2}(t)$ in the decomposition (1.3):

$$
X^{2}(t):=\left\{u_{0} \in C_{0}(\bar{\Omega}): u\left(\cdot, \tilde{t}, t, u_{0}\right) \text { has a zero in } \Omega \text { for all } \tilde{t}>t\right\} .
$$

The proofs of both Theorem 1.1 and (1.6) crucially depend on a new elliptic-type Harnack inequality, which was also established in [17] (see [17, Theorem 2.1]); we state this inequality in Theorem 3.7 below. By means of examples it has been shown in 13 , that there are bounded domains $\Omega$ such that, even if $L=\triangle$ (the Laplace operator), the following happens:

(i) Theorem 3.7 (with " $G=\Omega$ ") fails,

(ii) the inequality (1.5) fails for some $u$ and $v$ as in Theorem 1.1

(iii) (1.6) cannot be used as a characterization of the space $X^{2}(t)$ for which (1.3) and (1.4) hold.

Thus, according to (ii), on arbitrary bounded domains one cannot expect that signchanging solutions are exponentially dominated by positive solutions. In fact, the examples in 13 show that in general there are solutions which change sign for all times but become "asymptotically" positive, a phenomenon which does not occur on Lipschitz domains (see Proposition 6.11 and Remark 6.12). Therefore, as can be expected, the solutions of (1.1) on general bounded domains exhibit a more complicated behavior than those on Lipschitz domains, and the methods of [17] are not applicable here. All of the above mentioned difficulties have been overcome in [13] for divergence form equations. Among the basic tools we used there were the weak Green's function and the existence of a well defined adjoint equation. For the nondivergence form equations, however, we do not have these tools at our disposal and thus are faced with additional difficulties. Finally, unlike in the case of divergence form equations, where the basic solution space is "naturally" 
given, it is not at all obvious how one should define solutions of (1.1) on arbitrary bounded domains. The difficulties mentioned in this paragraph make the results to be presented particularly interesting and nontrivial.

In our main result, Theorem 2.2 below, we establish the existence of principal Floquet bundles and exponential separation under the sole assumption of boundedness of the spatial domain (assuming the coefficients in (1.1) satisfy (L1)). In particular, we give a new characterization of the space $X^{2}(t)$ in the decomposition (1.3). Instead of (1.6) (which we know may fail in general), we characterize it as the space of those initial conditions $u_{0}$ such that for all $T>t$ the corresponding solution $u\left(\cdot, \tilde{t} ; t, u_{0}\right)$ vanishes somewhere in $\bar{D}_{2}$ for some $\tilde{t} \geq T$, where $D_{2}$ is a fixed (sufficiently large) subdomain of $\Omega$ (and $D_{2}$ is independent of $u_{0}$ ). Another interesting point is that in Theorem 2.2 we establish a time-dependent decomposition of a time-dependent space of initial conditions; it is different from previous results of this kind where the space of initial conditions is always fixed (see (1.3)). That this space is time-dependent is a natural consequence of our choice of the definition of a solution of (1.1) (see Definition 4.7); this definition mimics in a "time-dependent" manner the definition introduced in 3 .

We present two applications of Theorem 2.2 Proposition 2.5 states the uniqueness of positive entire (defined for all $t \in \mathbb{R}$ ) solutions of (1.1). Next, as a special case of Theorem 2.2 assuming the coefficients of $L$ are time-periodic, we get a new result on the existence of a principal eigenvalue for time-periodic parabolic operators on nonsmooth domains (with nonsmooth coefficients). This application is treated in Corollary 2.6. This corollary also gives additional properties of the principal eigenvalue and the associated eigenfunction. In particular, we prove the existence of a uniform spectral gap between the principal eigenvalue and the rest of the spectrum for a class of time-periodic parabolic operators.

Similarly as in [3], an important feature of this paper is that in all our estimates the constants depend only on $N, \alpha_{0}, \beta_{0}$ in (L1) and on $\Omega$ but not on the smoothness of $\Omega$ (or the coefficients). From this standpoint, many of our results are new even if the domain is assumed smooth. Among the basic tools we use here are the maximum principle, the interior Harnack inequality, along with considerations of the behavior of solutions of (1.1) on "small" subdomains of $\Omega$. In this respect, we have greatly benefited from recent results established in [30] (see Lemma 3.5] and Theorem 3.6). Let us also point out that, despite the difficulties mentioned above, we do rely on the results established in [17. The general approach taken in this paper can be described as follows. We take a smooth subdomain $G$ of $\Omega$, which is close to $\Omega$ (in a suitable sense), and obtain appropriate estimates of solutions of (1.1) with " $\Omega=G$ " independently of the smoothness of $G$. Such estimates are obtained, in part, due to being able to control the behavior of solutions on small subdomains near the boundary $\partial G$. Once such estimates are established, we let $G$ converge to $\Omega$ in a suitable sense to get these estimates for the solutions of (1.1). Let us note that the above described strategy has also been used in 3 . Here, however, the matters are significantly more complicated by the time-dependent nature of the problem.

In this paper we only consider the Dirichlet problem. Related results for the oblique derivative problem can be found in 14, 24, 31, 33. Let us mention here that the techniques developed in this paper can also be applied to related problems on unbounded domains. Such results will appear elsewhere. 
The paper is organized as follows. Section 2 contains the statements of our main results. Section 3 contains some useful reference material, which will be used in later sections. In Section 4 we construct a function $w_{\Omega}$, which is used to define in what sense the solutions of (1.1) attain the boundary values. In Section 5 we prove Theorem 2.1 and establish some other properties of positive entire (defined for all $t \in \mathbb{R}$ ) solutions of (1.1). Sections 6 and 7 serve as a preparation for the proof of Theorem 2.2, More precisely, Section 6 is devoted to proving several estimates of positive solutions of (1.1); some of them might be of independent interest (see Theorem 6.1, Lemma 6.4, Proposition 6.11). Section7then contains some estimates of sign-changing solutions. Section 8 contains proofs of Theorem 2.2 and Corollary 2.4. Finally, Sections 9 and 10 contain proofs of Proposition 2.5] and Corollary 2.6. respectively.

\section{Statement of the MAin Results}

In this section and throughout the paper, whenever we refer to a solution of (1.1) we mean a solution as defined in Definition 4.7. We call a solution of (1.1) an entire solution if it is a solution of (1.1) on $\Omega \times \mathbb{R}$. The first result is the following theorem, which establishes the existence of a "well behaved" positive entire solution of (1.1).

Theorem 2.1. There exists a positive entire solution $\varphi_{\Omega}$ of (1.1) such that the following holds. If $D$ is a subdomain of $\Omega$ with $\bar{D} \subset \Omega$, then

$$
\frac{\varphi_{\Omega}(x, t)}{\left\|\varphi_{\Omega}(\cdot, t)\right\|_{L^{\infty}(\Omega)}} \geq C(D) \quad((x, t) \in D \times \mathbb{R}) .
$$

The constant $C(D)>0$ depends only on $D, \Omega$ and $N, \alpha_{0}$ and $\beta_{0}$ in $(L 1)$.

Our main result is the following theorem on exponential separation and principal Floquet bundles. Below we denote by $u\left(\cdot, t ; s, u_{0}\right)$ the solution of (1.1) with the initial condition $u(\cdot, s)=u_{0}$ (see Definition 4.7). The space $X(s)$ appearing in Theorem 2.2 is simply the space of all (appropriate) initial conditions $u(\cdot, s)=u_{0}$ such that the associated initial value problem has a solution $u\left(\cdot, t ; s, u_{0}\right)$ defined for all $t \geq s$ (for the precise definition, see (4.24)).

Theorem 2.2. The positive entire solution $\varphi_{\Omega}$ from Theorem 2.1 is uniquely determined by the condition (2.1) and by the assumption $\left\|\varphi_{\Omega}(\cdot, 0)\right\|_{L^{\infty}(\Omega)}=1$. There exists a subdomain $D_{2}$ of $\Omega$ with $\bar{D}_{2} \subset \Omega$ such that the following statements hold true.

(i) Let $X(s)$ be as in (4.24) and set

$$
\begin{aligned}
X^{1}(s) & :=\operatorname{span}\left\{\varphi_{\Omega}(\cdot, s)\right\}, \\
X^{2}(s) & :=\left\{u_{0} \in X(s): \text { for all } T>s \text { the function } u\left(\cdot, t ; s, u_{0}\right)\right. \\
& \text { has a zero in } \left.\bar{D}_{2} \text { for some } t \geq T\right\} .
\end{aligned}
$$

These sets are closed subspaces of $X(s)$. They are invariant under (1.1) in the following sense: if $i \in\{1,2\}, u_{0} \in X^{i}(s)$, then $u\left(\cdot, t ; s, u_{0}\right) \in X^{i}(t)$ $(t \geq s)$. Moreover, $X^{1}(s), X^{2}(s)$ are complementary subspaces of $X(s)$ :

$$
X(s)=X^{1}(s) \oplus X^{2}(s) \quad(s \in \mathbb{R}) .
$$


(ii) There are constants $C, \gamma>0$ depending only on $\Omega$ and $N, \alpha_{0}$ and $\beta_{0}$ in (L1) such that for any $u_{0} \in X^{2}(s)$ one has

$$
\frac{\left\|u\left(\cdot, t ; s, u_{0}\right)\right\|_{L^{\infty}(\Omega)}}{\left\|\varphi_{\Omega}(\cdot, t)\right\|_{L^{\infty}(\Omega)}} \leq C e^{-\gamma(t-s)} \frac{\left\|u_{0}\right\|_{L^{\infty}(\Omega)}}{\left\|\varphi_{\Omega}(\cdot, s)\right\|_{L^{\infty}(\Omega)}} \quad(t \geq s) .
$$

Remark 2.3. Let us point out that it follows from the proof of Theorem 2.2 that the domain $D_{2}$, whose existence is claimed, can in fact be any (smooth) domain $D_{2}$ with $\bar{D}_{2} \subset \Omega$ such that the (Lebesgue) measure $\left|\Omega \backslash \bar{D}_{2}\right|<\delta$, where $\delta>0$ depends only on $\Omega$ and $N, \alpha_{0}$ and $\beta_{0}$ in (L1).

We refer to the collection of the one-dimensional spaces $X^{1}(s), s \in \mathbb{R}$, as the principal Floquet bundle of (1.1) and to $X^{2}(s), s \in \mathbb{R}$, as its complementary Floquet bundle. Property (ii) as stated is an exponential separation between these two bundles. As discussed in the introduction, the existence of the Floquet bundles with exponential separation extends in a natural way the properties of the principal eigenvalue of time-independent (or time-periodic) parabolic problems. The positive entire solution $\varphi_{\Omega}$ serves as an analogue of the principal eigenfunction.

The following corollary gives an equivalent characterization of the space $X^{2}(s)$ in Theorem 2.2

Corollary 2.4. Let $X^{2}(s)(s \in \mathbb{R})$ and $D_{2}$ be as in Theorem 2.2. Then for any subdomain $D$ of $\Omega$ with $D_{2} \subseteq D \subset \bar{D} \subset \Omega$ the following holds:

$$
\begin{gathered}
X^{2}(s)=\left\{u_{0} \in X(s): \text { for all } T>s \text { the function } u\left(\cdot, t ; s, u_{0}\right)\right. \\
\text { has a zero in } \bar{D} \text { for some } t \geq T\} .
\end{gathered}
$$

As a consequence of the exponential separation estimate (2.3) we will derive the following uniqueness result.

Proposition 2.5. Let $\varphi_{1}, \varphi_{2}$ be positive entire solutions of (1.1) such that for some $c>0$ and $i=1,2$,

$$
\varphi_{i}(x, t) \leq c e^{c|t|} \quad((x, t) \in \Omega \times \mathbb{R}) .
$$

Then there exists a constant $q>0$ such that $\varphi_{1} \equiv q \varphi_{2}$.

The uniqueness of positive entire solutions has also been proved in 13, 15, 17, 25. 29, 31. We believe that the assumption (2.5) is not merely of a technical nature. We conjecture that (if $N \geq 2$ ) there are positive entire solutions, which grow superexponentially as time goes to negative infinity. We point out that the uniqueness results on smooth (at least Lipschitz) domains [15, 17, 25, 29, 31] exclude this possibility.

We now show another nontrivial application of Theorem 2.2. establishing the existence of a principal eigenvalue for a time-periodic parabolic eigenvalue problem. Suppose the coefficients of $L$ are $T$-periodic in $t$ for some $T>0$. Consider the eigenvalue problem

$$
\begin{aligned}
& u_{t}-L u=\lambda u \quad \text { in } \quad \Omega \times \mathbb{R}, \\
& u \stackrel{w_{\Omega}}{=} 0 \quad \text { on } \partial \Omega \times \mathbb{R}, \\
& u(\cdot, t)=u(\cdot, t+T) \quad \text { in } \quad \Omega \times \mathbb{R},
\end{aligned}
$$

where the boundary condition $u \stackrel{w_{\Omega}}{=} 0$ on $\partial \Omega \times \mathbb{R}$ is defined in Section 4 (it reduces to $u=0$ on $\partial \Omega \times \mathbb{R}$ if $\Omega$ is sufficiently regular). The principal eigenvalue $\lambda_{1}$ of 
this problem is the eigenvalue which is real and has a positive eigenfunction. The following corollary states the existence of such an eigenvalue for (2.6).

Corollary 2.6. Assume the coefficients of $L$ are $T$-periodic in $t$ for some $T>0$. Then there exists a unique eigenvalue $\lambda_{1}$ of (2.6) with the following properties.

(i) It is real and it has a unique (up to constant multiples) eigenfunction $\varphi_{1}$ which is positive in $\Omega \times \mathbb{R}$ and it satisfies the estimate (2.1).

(ii) $\left|\lambda_{1}\right|$ is bounded by a constant depending only on $\Omega$ and $N, \alpha_{0}$ and $\beta_{0}$ in $(L 1)$.

(iii) Let $\lambda \neq \lambda_{1}$ be any eigenvalue of (2.6) with a corresponding eigenfunction $\varphi_{\lambda}$. Then

$$
\operatorname{Re}(\lambda)-\lambda_{1} \geq \gamma>0,
$$

where $\gamma=\gamma\left(\Omega, N, \alpha_{0}, \beta_{0}\right)$ is as in (2.3). Moreover, if $\varphi_{\lambda}$ is real, then $\varphi(\cdot, \cdot)$ has a zero in $\bar{D}_{2} \times[t, t+T]$ for all $t \in \mathbb{R}$, where $D_{2}$ is as in Theorem 2.2 .

In the time-independent case, the existence of the principal eigenvalue for nondivergence form elliptic operators on general bounded domains has been proved in [3]. Even if we restrict ourselves to the autonomous case, Corollary 2.6 contains [3. Theorem 2.1] and provides an improvement of [3, Theorem 2.3]. In the timeperiodic case, our result is new in this generality. Indeed, for nondivergence form equations with periodic time-dependence the existence of the principal eigenvalue has been previously known only in the case of smooth coefficients and domain (see [11). For more general results of this kind for divergence form equations, we refer the reader to [7, 9].

\section{Preliminary Results}

Let $\Omega \subset \mathbb{R}^{N}$ be a fixed bounded domain and $L$ be an operator as in (1.2) with coefficients satisfying (L1). We will use the letter $G$ to denote a smooth (at least Lipschitz) subdomain of $\Omega$. For such a domain and an interval $J \subseteq \mathbb{R}$ consider a general linear problem

$$
\begin{aligned}
u_{t}-L u=0 & \text { in } \quad G \times J, \\
u=0 & \text { on } \quad \partial G \times J .
\end{aligned}
$$

Let us specify what notions of (super)solutions we use in this section. First, whenever we refer to (super)solutions of (3.1), we mean a function $u \in W_{\mathrm{loc}}^{2,1, N+1}(G \times J)$ such that (3.1) ((3.1) with "=" replaced by " $\geq$ ") is satisfied almost everywhere. Second, a (super)solution of (3.1), (3.2) is in addition continuous on $\bar{G} \times \bar{J}$ and satisfies (3.2) (3.2) with "=" replaced by " $\geq$ "). Below we often use standard maximum and comparison principles for (super)solutions of (3.1) as found in 21, for example.

The following results will be used frequently throughout the paper. To simplify the exposition we will make the following convention.

Convention. The statement "the constant $C$ depends on the structure of $L$ " means that $C$ is determined by the quantities $N, \alpha_{0}, \beta_{0}$ appearing in the condition (L1). Next, throughout the paper we often omit (some) arguments of the functions under consideration; for example, we use $u(t)$ instead of $u(\cdot, t)$ if there is no danger of confusion. Finally, whenever a constant $C>0$ may be assumed greater (or smaller) than 1 we assume it is such without further notice. For example, the 
constant $C$ in Theorem 3.1 below may be assumed greater than 1 and the constant $C_{2}(D, d)$ in Corollary 5.3 may be assumed less than 1 .

First, we state the interior Harnack inequality ([19]).

Theorem 3.1. Let $v$ be a nonnegative solution of $u_{t}-L u=0$ on $\Omega \times(0, \infty)$. Suppose $0<\delta<T$ and let $D$ be a subdomain of $\Omega$ such that $\operatorname{dist}(\bar{D}, \partial \Omega)=d>0$. Let $t, s \in \mathbb{R}$ be such that $s \geq \delta^{2}$, where $T \geq t-s \geq \delta^{2}$. Then there is a positive constant $C$ depending only on $\delta, T, D, d$ and the structure of $L$ such that one has

$$
\sup _{x \in D} v(x, s) \leq C \inf _{x \in D} v(x, t) .
$$

Consider now the following initial value problem:

$$
\begin{aligned}
u_{t}-L u=0 \quad \text { in } \quad & G \times(0, \infty), \\
u=0 \quad \text { on } \quad & \partial G \times(0, \infty), \\
u & =u_{0} \quad \text { in } \quad G \times\{0\},
\end{aligned}
$$

where $G$ is a smooth subdomain of $\Omega$. The following result is standard if $G$ is smooth. For Lipschitz $G$ it has been proved in [17.

Proposition 3.2. Let $G$ be a Lipschitz bounded domain. Assume $u_{0} \in C_{0}(\bar{G})$. Then the initial value problem (3.4) has a unique solution $u$ contained in $W_{\mathrm{loc}}^{2,1, p}(G \times(0, \infty)) \cap C(\bar{G} \times[0, \infty))$ for all $p>1$. It satisfies the following estimate:

$$
\|u(t)\|_{L^{\infty}(G)} \leq e^{m t}\left\|u_{0}\right\|_{L^{\infty}(G)} \quad(t \in[0, \infty))
$$

where $m=\sup _{G \times(0, \infty)}\left(c_{0}\right) \leq \beta_{0}\left(\beta_{0}\right.$ is as in $\left.(L 1)\right)$.

Remark 3.3. Obviously, there is nothing special about the interval $(0, \infty)$ in the above stated results. These results hold in the same way on any interval $(s, \infty)$ with $s \in \mathbb{R}$.

In the proof of existence of a principal Floquet bundle we will use in an essential way the following result of Varadhan (as attributed to him in [2]).

Lemma 3.4. Given any $a_{0}>0, b_{0} \geq 1$, there exists $\delta>0$ determined only by $a_{0}$, $b_{0}, N$, and $\operatorname{diam}(\Omega)$ such that for any closed set $K \subset \Omega$ with $|K| \leq \delta$ there exists a smooth function $g$ on $\Omega$ such that $1 \leq g \leq 2$ and for any symmetric positive definite matrix $\left(a_{i j}\right)$ with

one has

$$
\operatorname{det}\left(a_{i j}\right) \geq a_{0}^{N}
$$

For any $a \in \mathbb{R}$ we use the following notation throughout the paper:

$$
a^{+}=\max \{a, 0\}, \quad a^{-}=\max \{-a, 0\} .
$$

The next result (see [30, Lemma 3.1]) will be used frequently throughout the paper.

Lemma 3.5. Given any $k>0$ there is a constant $\delta>0$ determined only by the structure of $L, \operatorname{diam}(\Omega)$, and $k$ such that for any open set $U \subset \Omega$ with $|U|<\delta$ the following holds. If $v \in C(\bar{U} \times[\tau, T])$ is a supersolution of a problem (3.1), (3.2) with " $G=U$ " and $J=(\tau, T)$, then

$$
\left\|v^{-}(\cdot, t)\right\|_{L^{\infty}(U)} \leq 2 e^{-k(t-\tau)}\left\|v^{-}(\cdot, \tau)\right\|_{L^{\infty}(U)} \quad(t \in[\tau, T]) .
$$


Before we state the next result we need to introduce some notation. For a domain $D \subset \Omega$, we define the inner radius of $D$ to be

$$
\operatorname{inrad}(D):=\sup \left\{\rho>0: B\left(x_{0}, \rho\right) \subset D \text { for some } x_{0} \in D\right\},
$$

where $B\left(x_{0}, \rho\right)$ is the ball in $\mathbb{R}^{N}$ of radius $\rho$ centered at $x_{0}$. If $D_{0}$ and $D$ are bounded sets in $\mathbb{R}^{N}$, the notation $D_{0} \subset \subset D$ means $\bar{D}_{0} \subset D$. The following result will be crucial in our considerations; it has been proved in 30] (see Theorem 3.7 in [30] and the remarks following it). We state it in a simplified form tailored to our needs.

Theorem 3.6. Fix $\rho \in(0, \operatorname{diam}(\Omega) / 2)$. Then there exists $\delta$ determined only by $\rho$, $\operatorname{diam}(\Omega)$ and the structure of $L$ such that the following holds. Given any $d>0$, $\theta>0$, there exists a positive constant $\mu=\mu\left(N, \operatorname{diam}(\Omega), \alpha_{0}, \beta_{0}, d, \theta, \rho\right)$ with the following properties. If $D \subset G$ are subdomains of $\Omega$ satisfying

$$
\begin{aligned}
\operatorname{inrad}(D) & >\rho,|G \backslash \bar{D}|<\delta, \\
\operatorname{dist}(\bar{D}, \partial G) & >d,
\end{aligned}
$$

if $v \in C(\bar{G} \times[\tau, \infty))$ is a solution of a problem (3.1), (3.2) (with $J=(\tau, \infty)$ ), and if

$$
\begin{aligned}
v(x, t) & >0 \quad((x, t) \in \bar{D} \times[\tau, \tau+8 \theta)), \\
\left\|v^{-}(\cdot, \tau)\right\|_{L^{\infty}(G \backslash \bar{D})} & \leq \mu\|v\|_{L^{\infty}(D \times(\tau+\theta, \tau+2 \theta))},
\end{aligned}
$$

then the following statements hold true:

(s1) $v(x, t)>0((x, t) \in \bar{D} \times[\tau, \infty))$;

(s2) for any domain $H \subset \subset G$ with $H \cap D \neq \emptyset$ there is $T_{H}$ such that $v(\cdot, t)>0$ on $H$ for all $t \geq T_{H}$.

Finally, we state some results from [17]. First, a Harnack type inequality for quotients of positive solutions of (3.1), (3.2) (see [17, Theorem 2.1]).

Theorem 3.7. Let $G$ be a Lipschitz bounded domain. Let $\delta_{0}>0$ and let $u_{1}, u_{2}$ $\left(u_{2} \not \equiv 0\right)$ be two nonnegative solutions of (3.1), (3.2) on $G \times(0, \infty)$. Then for all $t \geq \delta_{0}$ the following estimate holds:

$$
\sup _{x \in G} \frac{u_{1}(x, t)}{u_{2}(x, t)} \leq C_{G} \inf _{x \in G} \frac{u_{1}(x, t)}{u_{2}(x, t)}
$$

with a constant $C_{G}>1$ depending only on $\delta_{0}$, the structure of $L$ and on the Lipschitz characteristics of $G$.

Recall that $u$ is an entire solution of (3.1), (3.2), if $u$ is a solution of (3.1), (3.2) on $G \times \mathbb{R}$. We then have the following theorem (see [17]).

Theorem 3.8. Let $G$ be a Lipschitz bounded domain. There exists a unique (up to constant multiples) positive entire solution $\varphi_{G}$ of (3.1), (3.2). Moreover, for every domain $D \subset \subset G$ there exists $C_{G}(D)>0$ such that

$$
\frac{\varphi_{G}(x, t)}{\left\|\varphi_{G}(\cdot, t)\right\|_{L^{\infty}(G)}} \geq C_{G}(D) \quad((x, t) \in D \times \mathbb{R}) .
$$

The constant $C_{G}(D)$ depends only on D, the structure of $L$ and on the Lipschitz characteristics of $G$. 


\section{Construction of $w_{\Omega}$}

Before we proceed to prove the existence of a positive entire solution of (1.1) we need to define first what we mean by a solution of (1.1); most importantly, we need to explain in what sense the solutions of (1.1) satisfy the boundary condition on $\partial \Omega$ if the boundary $\partial \Omega$ is "rough". We cannot require, in general, that the zero boundary values be attained (continuously) at all points of the boundary.

Let us recall here the approach used in 3 . In that paper, assuming the coefficients of $L$ in (1.2) (and hence of $M$ ) are time-independent, the authors proved the existence of a bounded positive solution $w_{0}$ of

$$
\begin{array}{rll}
-M u=1 & \text { in } & \Omega, \\
u=0 & \text { on } & \partial \Omega,
\end{array}
$$

which attains the boundary values in some sense on $\partial \Omega$. Then, for any $f \in L^{N}(\Omega)$, a solution of $L u=f$ in $\Omega, u=0$ on $\partial \Omega$ is defined to be a function $u \in W_{l o c}^{2, N}(\Omega)$, for which $L u=f$ almost everywhere in $\Omega$ and the boundary condition is attained in the following sense:

$$
u \stackrel{w_{0}}{=} 0
$$

which means that $u\left(x_{j}\right) \rightarrow 0$ for all sequences $x_{j} \rightarrow \partial \Omega$ such that $w_{0}\left(x_{j}\right) \rightarrow 0$. It has also been proved in [3] that $w_{0}$ attains the zero boundary value continuously at each point $y \in \partial \Omega$ which admits a strong barrier; the definition of a strong barrier at a boundary point is as follows.

Definition 4.1. A point $y \in \partial \Omega$ is said to admit a strong barrier if for some ball $B(y, r)$ there is in $B(y, r) \cap \Omega=V$ a positive function $h \in W_{l o c}^{2, N}(V)$ satisfying $M h \leq-1$, which can be extended continuously to the point $y$ with $h(y)=0$.

Remark 4.2. K. Miller [28] has shown that any point $y \in \partial \Omega$ at which $\partial \Omega$ satisfies an exterior cone condition admits a strong barrier $h \in C^{2}(V)$ such that $M h \leq-1$ on $V$ for all operators $M$ whose coefficients satisfy (L1); here $V$ is some fixed relative neighborhood of $y$ in $\Omega$.

Here we will adopt a similar "time-dependent" approach. Our goal is to prove the existence of a positive function $w_{\Omega} \in \bigcap_{p>1} W_{l o c}^{2,1, p}(\Omega \times \mathbb{R}) \cap C(\Omega \times \mathbb{R}) \cap L^{\infty}(\Omega \times \mathbb{R})$ which satisfies (with $M$ as in (1.2))

$$
\begin{aligned}
\left(w_{\Omega}\right)_{t}-M w_{\Omega}=1 & \text { in } \quad \Omega \times \mathbb{R}, \\
w_{\Omega}=0 & \text { on } \quad \partial \Omega \times \mathbb{R},
\end{aligned}
$$

and the boundary values are attained in a similar sense as for the function $w_{0}$ above. The function $w_{\Omega}$ will then be used in Definition 4.7 of solutions of (1.1).

To prove the existence of $w_{\Omega}$ we use a limiting procedure. Let $G \subset \subset \Omega$ be a smooth domain and let $T>0$. Consider the following problem:

$$
\begin{array}{rll}
u_{t}-M u=1 & \text { in } \quad G \times(-T, \infty), \\
u=0 & \text { on } \quad(\partial G \times(-T, \infty)) \cup(G \times\{-T\}) .
\end{array}
$$

By [21, Theorem 7.17] this problem has a unique solution $w_{G, T} \in \bigcap_{p>1}$ $W^{2,1, p}(G \times(-T, T)) \cap C(\bar{G} \times[-T, T])$, which is in addition positive by the maximum principle. Let us prove a uniform bound on $w_{G, T}$ independent of $G \subset \subset \Omega$ 
and $T>0$. Without loss of generality we may assume $\inf _{x=\left(x_{1}, \ldots, x_{N}\right) \in \Omega} x_{1}=0$. Define the function

$$
u_{G, T}=e^{\left(\left(1+\beta_{0}\right) / \alpha_{0}\right) \operatorname{diam}(\Omega)}-e^{\left(\left(1+\beta_{0}\right) / \alpha_{0}\right) x_{1}}-w_{G, T} .
$$

A simple calculation shows that $\left(u_{G, T}\right)_{t}-M u_{G, T} \geq 0$ on $G \times(-T, \infty), u_{G, T} \geq 0$ on $(\partial G \times(-T, \infty)) \cup(G \times\{-T\})$. The maximum principle then implies that $u_{G, T} \geq 0$ on $G \times(-T, \infty)$. We have thus proved

$$
0<w_{G, T}(x, t) \leq e^{\left(\left(1+\beta_{0}\right) / \alpha_{0}\right) \operatorname{diam}(\Omega)} \quad((x, t) \in G \times(-T, \infty)),
$$

whenever $G \subset \subset \Omega$ is smooth and $T>0$. The inequality (4.8) and standard parabolic regularity estimates imply that if $T_{i} \rightarrow \infty$, then, at least for a subsequence, $w_{G, T_{i}} \rightarrow w_{G}$ uniformly on compact subsets of $\bar{G} \times \mathbb{R}, w_{G} \in \bigcap_{p>1}$ $W_{l o c}^{2,1, p}(G \times(-T, T)) \cap C(\bar{G} \times[-T, T])$ for all $T>0$, and $w_{G}$ satisfies

$$
\begin{aligned}
\left(w_{G}\right)_{t}-M w_{G}=1 & \text { in } \quad G \times \mathbb{R}, \\
w_{G}=0 & \text { on } \quad \partial G \times \mathbb{R},
\end{aligned}
$$

and

$$
0<w_{G}(x, t) \leq e^{\left(\left(1+\beta_{0}\right) / \alpha_{0}\right) \operatorname{diam}(\Omega)} \quad((x, t) \in G \times \mathbb{R}) .
$$

Let us now prove the uniqueness of $w_{G}$ in case $G$ is smooth.

Proposition 4.3. If $G \subset \subset \Omega$ is smooth, then there exists a unique $w_{G}$ satisfying (4.9), (4.10), (4.11). More generally, for any bounded domain $G$, if $w_{1}$ and $w_{2}$ are bounded solutions of (4.9), (4.10) such that $w_{i} \in C(\bar{G} \times \mathbb{R}), i=1,2$, then $w_{1} \equiv w_{2}$.

Proof. Since we have already proved the existence of $w_{G}$ it remains to prove the uniqueness statement. Let $w_{1}, w_{2}$ be as in the statement of the proposition. For $a>1$ set $v_{a}=a w_{1}-w_{2}$. The boundedness of $w_{i}, i=1,2$, implies that there exists $\epsilon_{a}>0$ such that $\left(v_{a}\right)_{t}-M v_{a}=a-1 \geq-\epsilon_{a} v_{a}$ on $G \times \mathbb{R}$; by continuity of $w_{1}, w_{2}$, we also have $v_{a}=0$ on $\partial G \times \mathbb{R}$. By the maximum principle we get

$$
\left\|v_{a}^{-}(\cdot, t)\right\|_{L^{\infty}(G)} \leq e^{-\epsilon_{a}(t-\tau)}\left\|v_{a}^{-}(\cdot, \tau)\right\|_{L^{\infty}(G)} \quad(t \geq \tau) .
$$

Sending $\tau \rightarrow-\infty$ and using the boundedness of $v_{a}$ we obtain $v_{a}^{-} \equiv 0$; hence $a w_{1} \geq w_{2}$. Since this is true for any $a>1$ we get $w_{1} \geq w_{2}$. By symmetry of the argument also $w_{2} \geq w_{1}$ and therefore $w_{1} \equiv w_{2}$. Finally, if $G$ is smooth, then any bounded solution $w$ of (4.9), (4.10) belongs to $C(\bar{G} \times \mathbb{R})$ and hence has to be identically equal to $w_{G}$, completing the proof.

Next, we give a monotonicity result for $w_{G}$.

Proposition 4.4. Let $G_{1} \subseteq G_{2}$ be two smooth bounded domains and let $w_{G_{1}}$, $w_{G_{2}}$ be the corresponding solutions given by Proposition 4.3. Then $w_{G_{1}} \leq w_{G_{2}}$ on $G_{1} \times \mathbb{R}$.

Proof. Fix $a>1$. Then the function $\tilde{v}_{a}=a w_{G_{2}}-w_{G_{1}}$ is bounded and hence, for some $\epsilon_{a}>0$, we have $\left(\tilde{v}_{a}\right)_{t}-M \tilde{v}_{a}=a-1 \geq-\epsilon_{a} v_{a}$ on $G_{1} \times \mathbb{R}$ and also $\tilde{v}_{a}=a w_{G_{2}} \geq 0$ on $\partial G_{1} \times \mathbb{R}$. By the maximum principle,

$$
\left\|\tilde{v}_{a}^{-}(\cdot, t)\right\|_{L^{\infty}\left(G_{1}\right)} \leq e^{-\epsilon_{a}(t-\tau)}\left\|\tilde{v}_{a}^{-}(\cdot, \tau)\right\|_{L^{\infty}\left(G_{1}\right)} \quad(t \geq \tau) .
$$

The boundedness of $\tilde{v}_{a}$ then implies $\tilde{v}_{a}^{-} \equiv 0$ on $G_{1} \times \mathbb{R}$; therefore $a w_{G_{2}} \geq w_{G_{1}}$. Since this is true for any $a>1$, we get $w_{G_{2}} \geq w_{G_{1}}$. 
We are ready to construct the solution $w_{\Omega}$. Let $\Omega_{j} \subset \subset \Omega_{j+1} \subset \subset \Omega$ be a sequence of smooth subdomains such that $\bigcup_{j=1}^{\infty} \Omega_{j}=\Omega$, and let $w_{\Omega_{j}}$ be the corresponding solutions given by Proposition 4.3. The uniform bound and monotonicity of $w_{\Omega_{j}}$ with respect to $j$ and standard parabolic regularity imply that there exists a function $w_{\Omega}=\lim _{j \rightarrow \infty} w_{\Omega_{j}}, w_{\Omega} \in \bigcap_{p>1} W_{l o c}^{2,1, p}(\Omega \times \mathbb{R}) \cap C(\Omega \times \mathbb{R})$, and (4.9) and (4.11) are satisfied with $G$ replaced by $\Omega$. Moreover, $w_{\Omega}$ vanishes continuously at each point $y \in \partial \Omega$ where $\partial \Omega$ satisfies an exterior cone condition. To prove this fact one can use Remark 4.2 and arguments very similar to those in 3 (see the discussion preceding [3, Remark 3.1]), and we omit the details.

Remark 4.5. The above defined function $w_{\Omega}$ is independent of the approximating sequence $\Omega_{j}$. Indeed, this follows immediately from Proposition 4.4.

The next proposition states that the boundary behavior of $w_{\Omega}$ depends only on a neighborhood of $\partial \Omega$ (cf. [3, Lemma 3.1]).

Proposition 4.6. Let $G_{1}, G_{2}$ be smooth domains such that $G_{1} \subset \subset G_{2} \subset \subset \Omega$. Then there exists a constant $C=C\left(G_{1}, G_{2}, \Omega\right)>0$, depending only on $G_{1}, G_{2}, \Omega$ and on the structure of $L$, such that

$$
w_{\Omega \backslash \bar{G}_{1}}(x, t) \leq w_{\Omega}(x, t) \leq C w_{\Omega \backslash \bar{G}_{1}}(x, t) \quad\left((x, t) \in\left(\Omega \backslash \bar{G}_{2}\right) \times \mathbb{R}\right),
$$

where $w_{\Omega \backslash \bar{G}_{1}}$ is the function as constructed above but corresponding to $\Omega \backslash \bar{G}_{1}$.

Proof. We note first that the existence of $w_{\Omega}$ can be established for any bounded open set $\Omega$ by repeating the above arguments for each connected component of $\Omega$. Therefore the function $w_{\Omega \backslash \bar{G}_{1}}$ is well defined. Similarly, for Proposition 4.4 to hold we only need that $G_{1}, G_{2}$ in that proposition be bounded open sets with $G_{1} \subseteq G_{2}$. This implies the first inequality in (4.13).

We now prove the remaining inequality. Let $\Omega_{j}, j \geq 1$, be a strictly increasing sequence of smooth subdomains of $\Omega$ such that their union is equal to $\Omega$. We may assume that all of these domains contain $G_{2}$ and $\operatorname{dist}\left(\bar{G}_{2}, \partial \Omega_{j}\right) \geq \operatorname{dist}\left(\bar{G}_{2}, \partial \Omega\right) / 2$ for all $j \geq 1$. We have $w_{\Omega_{j} \backslash \bar{G}_{1}}(x, t) \geq \tilde{C}>0$ for all $x \in \partial G_{2}, t \in \mathbb{R}, j \geq 1$ and a constant $\tilde{C}$ depending only on $G_{1}, G_{2}, \Omega$ and the structure of $L$. This fact follows from a result of Krylov and Safonov (see 20, Section 4.1, Lemma 1 and Theorem $2]$, for example). Therefore, using the boundary condition and boundedness of $w_{\Omega_{j}}$, we have $v_{j}=a w_{\Omega_{j} \backslash \bar{G}_{1}}-w_{\Omega_{j}} \geq 0$ on $\partial\left(\Omega_{j} \backslash \bar{G}_{2}\right) \times \mathbb{R}$ for all $j \geq 1$ and some sufficiently large $a>1$ depending only on $G_{1}, G_{2}, \Omega$ and the structure of $L$. The same arguments as in the proof of Proposition 4.3 (see 4.12) show that $v_{j} \geq 0$ on $\left(\Omega_{j} \backslash \bar{G}_{2}\right) \times \mathbb{R}$. Letting $j \rightarrow \infty$, we obtain the right hand side inequality in (4.13).

We will now define what we mean by a solution of (1.1) (or an associated initial value problem) for a general bounded domain $\Omega$. First, we say that

$$
u \stackrel{w_{\Omega}}{=} 0 \quad \text { on } \partial \Omega \times\left[t_{0}, t_{1}\right],
$$

if $u\left(x_{j}, t_{j}\right) \rightarrow 0$ for all sequences $x_{j} \rightarrow \partial \Omega$ and $t_{j} \in\left[t_{0}, t_{1}\right]$ such that $w_{\Omega}\left(x_{j}, t_{j}\right) \rightarrow 0$. Let $Y=C(\Omega) \cap L^{\infty}(\Omega)$ be endowed with the supremum norm. Then $Y$ is a closed subspace of $L^{\infty}(\Omega)$. Define the set

$$
Y(s):=\left\{u_{0} \in C(\Omega) \cap L^{\infty}(\Omega): u_{0} \stackrel{w_{\Omega}}{=} 0 \text { on } \partial \Omega \times[s]\right\} \quad(s \in \mathbb{R}) .
$$

Endowing $Y(s)$ with the supremum norm it is easy to prove that it is a closed subspace of $Y$. 
Definition 4.7. Let $s<T, s, T \in \mathbb{R}$, and let $u_{0} \in Y(s)$. A function $u$ is a solution of the initial value problem

$$
\begin{aligned}
u_{t}-L u=0 \quad \text { in } \quad & \Omega \times(s, T), \\
u=0 \quad \text { on } \quad & \partial \Omega \times(s, T), \\
u=u_{0} \quad \text { in } \quad & \Omega \times\{s\},
\end{aligned}
$$

if $u \in W_{l o c}^{2,1, N+1}(\Omega \times(s, T)) \cap C(\Omega \times[s, T)) \cap L^{\infty}(\Omega \times(s, T))$, $u$ satisfies $u_{t}-L u=0$ almost everywhere in $\Omega \times(s, T)$, and $u \stackrel{w_{\Omega}}{=} 0$ on $\partial \Omega \times[s, \tau]$ for all $\tau \in[s, T)$. Further, a function $u$ is a solution of (1.1) if $u$ is a solution of an initial value problem (4.16) for all $s<T$ with $s, T \in J$.

Remark 4.8. Proposition 4.6 shows that the boundary condition (4.14) and thus also Definition 4.7 depends only on a neighborhood of the boundary $\partial \Omega$. If $\Omega$ is sufficiently regular, say, Lipschitz or, more generally, satisfying an exterior cone condition at each $y \in \partial \Omega$, then $Y(s)=C_{0}(\bar{\Omega})$ for all $s \in \mathbb{R}$ and Definition 4.7 reduces to the usual definition of a (strong) solution of (1.1).

The following example illustrates that the boundary condition (4.14) used in our definition of solutions of (1.1) can in general be time-dependent.

Example. Let us demonstrate that, unlike the case of smooth domains, the attainment of $w_{\Omega}(\cdot, t)$ of the boundary values on $\partial \Omega$ may depend substantially on $t \in \mathbb{R}$. For any $N \geq 2$ let $\Omega=B_{1}-\{0\}$, a punctured unit ball in $\mathbb{R}^{N}$ centered at the origin. Define the coefficients of the operator $L$ to be

$$
a_{i j}(x, t)=\delta_{i j}+g(t) \frac{x_{i} x_{j}}{|x|^{2}}, b_{i} \equiv 0, c_{0} \equiv 0 \quad((x, t) \in \Omega \times \mathbb{R}),
$$

where $g(t) \equiv N-1$ if $t \leq 1-N, g(t)=-t$ for $t \in[1-N, 0]$, and $g(t) \equiv 0$ if $t>0$. Then $L$ satisfies (L1) with $\alpha_{0}=1$ and $\beta_{0}=N$. Let $w_{0}$ be the function satisfying (4.1), (4.2) with $M$ equal to the operator defined in (4.17) with $t \leq 1-N$, which we denote for now by $M_{0}$. It is not difficult to see that then $w_{\Omega}(\cdot, t) \equiv w_{0}$ for all $t \leq 1-N$. Indeed, this can be proved using the fact that $w_{\Omega}$ and $w_{0}$ are obtained as the (monotone) limits of suitable solutions on smooth subdomains $\Omega_{j}$ converging to $\Omega$. Next, a simple computation shows that $\tilde{u}(x)=1-|x|^{1 / N}$ is a positive smooth bounded function on $\Omega$, vanishing on $\partial B_{1}$, and satisfying $M_{0} \tilde{u}=0$ in $\Omega$. The existence of $\tilde{u}$, the definition of the refined maximum principle (see [3]) and [3, Lemma 3.2] imply that there exists a sequence $x_{j} \rightarrow 0$ such that $w_{0}\left(x_{j}\right)=w_{\Omega}\left(x_{j}, t\right) \rightarrow 0(t \leq 1-N)$. On the other hand, for $t>0, w_{\Omega}$ is a positive bounded solution of $u_{t}-\Delta u=1$ on a punctured ball and thus (see [8]) can be uniquely extended to a solution of the same equation on $B_{1}$. Therefore, for all $t>0$, we have $w(x, t) \geq \alpha(t)>0$ on a neighborhood of the origin, which shows that, in general, the boundary behavior of $w_{\Omega}(\cdot, t)$ may change substantially in $t$ (see also Remark 4.14). In particular, for $s>0$ the solutions of the initial value problem (4.16) exist for all $u_{0} \in C_{0}\left(\bar{B}_{1}\right)=Y(s)$, whereas for $s \leq 1-N$ we have $Y(s) \subsetneq C_{0}\left(\bar{B}_{1}\right)$.

Let us conclude this section with a discussion of properties of solutions of (1.1) as defined in Definition 4.7. We first show that they still satisfy a modified version of the comparison principle. In the following proposition we say

$$
\liminf u \stackrel{w_{\Omega}}{\geq} 0 \quad \text { on } \partial \Omega \times[s, T)
$$


if for any $\tau \in[s, T)$ we have $\liminf u\left(x_{j}, t_{j}\right) \geq 0$ for all sequences $x_{j} \rightarrow \partial \Omega$ and $t_{j} \in[s, \tau]$ such that $w_{\Omega}\left(x_{j}, t_{j}\right) \rightarrow 0$.

Proposition 4.9. Let $u_{0} \geq 0$ in $\Omega$, and suppose that $u \in W_{l o c}^{2,1, N+1}(\Omega \times(s, T)) \cap$ $C(\Omega \times[s, T)), u(\cdot, s)=u_{0}, u$ is bounded below on $\Omega \times(s, \tau)$ for each $\tau<T$, and

$$
\begin{array}{cc}
u_{t}-L u \geq 0 & \text { in } \Omega \times(s, T), \\
\liminf u \stackrel{w_{\Omega}}{\geq} 0 & \text { on } \partial \Omega \times[s, T) .
\end{array}
$$

Then $u \geq 0$ on $\Omega \times(s, T)$.

Remark 4.10. By [3, Remark 1.1] the requirement that $u$ be bounded below cannot be omitted.

Proof of Proposition 4.9. Without loss of generality we will assume in this proof that $c_{0} \leq 0$. Fix $\tau \in[s, T)$ and denote $A=\sup _{\Omega \times(s, \tau)} u^{-}$. A simple contradiction argument using (4.20) shows that for each $\epsilon>0$ there exists $\delta(\epsilon)>0$ such that

$$
u \geq-\epsilon-\frac{A}{\delta(\epsilon)} w_{\Omega} \quad \text { on } \Omega \times[s, \tau] .
$$

Now fix $x_{0} \in \Omega$ and let $x_{0} \in \Omega_{j} \subset \subset \Omega_{j+1} \subset \subset \Omega$ be a sequence of smooth subdomains such that $\bigcup_{j=1}^{\infty} \Omega_{j}=\Omega$. Let $w_{\Omega_{j}}$ be as given by Proposition 4.3. Define

$$
v=u+\epsilon+\frac{A}{\delta(\epsilon)}\left(w_{\Omega}-w_{\Omega_{j}}\right) \quad \text { on } \Omega_{j} \times[s, \tau] .
$$

Using (4.21) and the fact that $w_{\Omega_{j}}=0$ on $\Omega_{j} \times \mathbb{R}$, we get $v \geq 0$ on $\partial \Omega_{j} \times$ $[s, \tau]$. Moreover, by the assumption $u_{0} \geq 0$ and by the monotonicity of $w_{\Omega}$ with respect to domain inclusion (Proposition 4.4), we also have $v \geq 0$ on $\Omega_{j} \times\{s\}$. A straightforward computation, employing $c_{0} \leq 0$ and Proposition 4.4, shows that $v_{t}-L v \geq 0$ on $\Omega_{j} \times(s, \tau)$. By the usual comparison principle, we then have $v \geq 0$ on $\Omega_{j} \times[s, \tau]$. This fact leads, upon letting $j \rightarrow \infty$, to the conclusion that $u\left(x_{0}, t\right) \geq-\epsilon$ for all $t \in[s, \tau]$ and all $\epsilon>0$. Therefore $u \geq 0$ on $\Omega \times(s, \tau)$ and since $\tau<T$ was arbitrary the proof is complete.

As an immediate consequence we obtain the following corollary.

Corollary 4.11. Let $u$ be a solution of (4.16). Then

$$
\|u(t)\|_{L^{\infty}(\Omega)} \leq e^{m(t-s)}\left\|u_{0}\right\|_{L^{\infty}(\Omega)} \quad(t \in[s, T]),
$$

where $m=\sup _{\Omega \times(s, T)}\left(c_{0}\right) \leq \beta_{0}\left(\beta_{0}\right.$ is as in (L1)). In particular, the solutions of (4.16) are unique (if they exist).

Next, with $Y(s)$ as defined in (4.15), set

$$
X(s):=\left\{u_{0} \in Y(s): \text { (4.16) has a solution for all } T>s\right\} \quad(s \in \mathbb{R}) .
$$

Corollary 4.11 together with standard parabolic regularity estimates implies that if $X(s)$ is endowed with the supremum norm, then it becomes a closed subspace of $Y(s)$.

Remark 4.12. We note, similarly as in Remark 4.8, that if $\Omega$ is sufficiently regular, then $X(s)=Y(s)=C_{0}(\bar{\Omega}), s \in \mathbb{R}$. However, in the example appearing after Remark 4.8 one has $X(s)=Y(s)=C_{0}\left(\bar{B}_{1}\right)$ for $s>0$ and $X(s) \subseteq Y(s) \subsetneq C_{0}\left(\bar{B}_{1}\right)$ whenever $s \leq 1-N$, and thus the spaces $X(s), Y(s)$ in general do depend nontrivially on $t$. 
Let $\Omega_{j} \subset \subset \Omega_{j+1} \subset \subset \Omega$ be a sequence of smooth subdomains such that $\bigcup_{j=1}^{\infty} \Omega_{j}=$ $\Omega$. For any integer $j \geq 2$ let $\xi_{j}$ be a smooth function compactly supported in $\Omega_{j}$ such that $\xi_{j} \equiv 1$ on $\Omega_{j-1}$ and $0 \leq \xi_{j} \leq 1$. The following result will be useful in the sequel.

Proposition 4.13. Let $\xi_{j}, \Omega_{j}$ be as above. Let $u_{0} \in X(s)$ and let $u$ be the corresponding solution of (4.16). Let $u_{j}$ be the solution of (4.16) with $\Omega$ replaced by $\Omega_{j}$ and with $u_{0}$ replaced by $\xi_{j} u_{0}$. Then (a subsequence of) the sequence $u_{j}$ converges uniformly to $u$ on compact subsets of $\Omega \times[s, \infty)$.

Proof. Without loss of generality suppose $c_{0} \leq 0$ in this proof. Note that by Proposition 3.2 and standard parabolic regularity (a subsequence of) the sequence $u_{j}$ converges to some $\tilde{u}$ locally uniformly on $\Omega \times(s, \infty)$ and $\tilde{u} \in \bigcap_{p>1} W_{l o c}^{2,1, p}$ $(\Omega \times(s, \infty)) \cap C(\Omega \times(s, \infty)), \tilde{u}_{t}-L \tilde{u}=0$ in $\Omega \times(s, \infty)$, and $\tilde{u}$ is bounded on $\Omega \times(s, T)$ for all $T>s$. The definition of $\xi_{j}$ implies that if $m \geq n$, then $u_{m}(\cdot, s)-u_{n}(\cdot, s) \equiv 0$ on $\Omega_{n-1}$. Employing the same barrier arguments as, for example, in the proof of [17. Proposition 5.4], one can prove that in fact (passing to a subsequence) the $u_{j}$ converge uniformly to $\tilde{u}$ on compact subsets of $\Omega \times[s, \infty)$. Thus $\tilde{u} \in C(\Omega \times[s, \infty))$ and $\tilde{u}(x, s)=\lim _{j \rightarrow \infty} u_{j}(x, s)=\lim _{j \rightarrow \infty} \xi_{j}(x) u_{0}(x)=u_{0}(x)$ for all $x \in \Omega$. To conclude that $\tilde{u}=u$ we still need to show that $\tilde{u} \stackrel{w_{\Omega}}{=} 0$ on $\partial \Omega \times[s, T]$ for all $T>s$.

We denote $A=\left\|u_{0}\right\|_{L^{\infty}(\Omega)}$. The definition of $\xi_{j}$ and a simple contradiction argument show that for each $\epsilon>0$ there exists $\delta(\epsilon)>0$ such that for each $j$ we have

Set

$$
\left|u_{j}(x, s)\right| \leq\left|u_{0}(x)\right| \leq \epsilon+\frac{A}{\delta(\epsilon)} w_{\Omega}(x, s) \quad\left(x \in \Omega_{j}\right)
$$

$$
v(x, t)=\epsilon+\frac{A}{\delta(\epsilon)} w_{\Omega}(x, t)-u_{j}(x, t) \quad\left((x, t) \in \Omega_{j} \times[s, \infty)\right) .
$$

Then an easy computation shows that $v_{t}-L v \geq 0$ on $\Omega_{j} \times(s, \infty)$ and $v$ is nonnegative on the parabolic boundary of that set. The usual comparison principle then gives that $v \geq 0$ on $\Omega_{j} \times[s, \infty)$. The same conclusion can be reached if the minus sign in the definition of $v$ is replaced by the plus sign. As a consequence we obtain that for each $\epsilon>0$ there exists $\delta(\epsilon)>0$ such that

$$
|\tilde{u}(x, t)|=\lim _{j \rightarrow \infty}\left|u_{j}(x, t)\right| \leq \epsilon+\frac{A}{\delta(\epsilon)} w_{\Omega}(x, t) \quad((x, t) \in \Omega \times[s, \infty)) .
$$

Obviously, (4.25) implies $\tilde{u} \stackrel{w_{\Omega}}{=} 0$ on $\partial \Omega \times[s, T]$ for all $T>s$. Thus, by Corollary 4.11, we must have $\tilde{u} \equiv u$.

Remark 4.14. We remark that if $u_{0}$ is smooth and compactly supported in $\Omega$ we may take $\xi_{j} \equiv 1\left(\right.$ on $\Omega_{j}$ ) in the above proof, showing that $u_{0} \in X(s), s \in \mathbb{R}$. However, smooth functions compactly supported in $\Omega$ may not be dense in $X(s)$; this situation occurs (for any $s>0$ ) in the example appearing after Remark 4.8 (see also Remark 4.12).

\section{Existence of A POSitive EntiRe SOLUtion}

In this section we prove the existence of a "well behaved" positive entire solution $\varphi_{\Omega}$ of (1.1). We will obtain $\varphi_{\Omega}$ as the limit of a (sub)sequence of positive entire solutions $\varphi_{\Omega_{j}}$ given by Theorem 3.8 with " $G=\Omega_{j}$ ", where $\Omega_{j}$ is a sequence of smooth subdomains of $\Omega$ converging (in the Hausdorff metric) to $\Omega$. To be able 
to pass to the limit we need an estimate as in (3.14) with $C_{G}$ independent of the smoothness of $G$. We establish such an estimate in Lemma 5.2 below. Before we state it let us introduce some notation.

In the remainder of this paper we fix

$$
x_{\Omega} \in \Omega \text { with } \operatorname{dist}\left(x_{\Omega}, \partial \Omega\right)=\operatorname{inrad}(\Omega), \quad c_{\Omega} \geq 1,
$$

where $\operatorname{inrad}(\Omega)$ is defined in (3.9) and $c_{\Omega}$ is the constant in Theorem 3.1 with " $D=B\left(x_{\Omega}, \operatorname{inrad}(\Omega) / 4\right) ", " \Omega=B\left(x_{\Omega}, \operatorname{inrad}(\Omega) / 2\right) ", " \delta=1 ", " T=2 "$. Note that $c_{\Omega}$ depends only on $\operatorname{inrad}(\Omega)$ and the structure of $L$, and we can assume without loss of generality that $c_{\Omega} \geq 1$. With $\alpha_{0}, \beta_{0}$ as in (L1), set $a_{0}=\alpha_{0}$ and $b_{0}=3\left(\sqrt{N} \beta_{0}+\log c_{\Omega}\right)$ in Lemma 3.4 and let $\delta=\delta_{0}>0$ be as in that lemma. The constant $\delta_{0}$ depends only on $\operatorname{inrad}(\Omega), \operatorname{diam}(\Omega)$, and the structure of $L$. Now choose a smooth domain $D_{0}$ such that

$$
B\left(x_{\Omega}, \operatorname{inrad}(\Omega) / 2\right) \subseteq D_{0} \subset \subset \Omega \text { and }\left|\Omega \backslash D_{0}\right|<\delta_{0} .
$$

From now on we will work with this $D_{0}$.

Remark 5.1. In addition to $D_{0}$ chosen above, in the course of this paper we fix (smooth) domains $D_{i}, i=1,2$ such that $D_{i} \subset \subset D_{i+1} \subset \subset \Omega, i=0,1,2$. As we shall see, their choice is only determined by requiring that $\left|\Omega \backslash D_{i}\right|<\delta$, where $\delta>0$ is a number depending only on $\Omega$ and the structure of $L$. Thus any dependence expressed in terms of $D_{i}$ (or quantities related to it, such as $d_{i}=\operatorname{dist}\left(\bar{D}_{i}, \partial \Omega\right)$ ) can be stated as a dependence on $\Omega$ and the structure of $L$ only. We use this observation without further notice.

Lemma 5.2. Let $D_{0}$ be as in (5.2). Let $D, G$ be domains such that $G$ is smooth and $D_{0} \subseteq D \subset \subset G \subseteq \Omega$. Then, if $\operatorname{dist}(\bar{D}, \partial G) \geq d>0$, we have

$$
\frac{\varphi_{G}(x, t)}{\left\|\varphi_{G}(\cdot, t)\right\|_{L^{\infty}(G)}} \geq C_{1}(D, d) \quad((x, t) \in D \times \mathbb{R}),
$$

where $\varphi_{G}$ is as in Theorem 3.8. The constant $C_{1}(D, d)>0$ depends only on $\operatorname{inrad}(\Omega), D, d$, and the structure of $L$.

Proof. Let $D, G, \varphi_{G}$ be as in the statement of the lemma and let $D_{0}$ be as in (5.2). Fix $t_{0} \in \mathbb{R}$. By the Harnack inequality (3.3) we have for any $t \leq t_{0}-2$,

$$
\begin{aligned}
\sup _{x \in D} \varphi_{G}(x, t) & \leq C \varphi_{G}\left(x_{\Omega}, t+1\right) \leq \cdots \leq C c_{\Omega}^{\left[t_{0}-t-2\right]} \varphi_{G}\left(x_{\Omega}, t+\left[t_{0}-t-2\right]+1\right) \\
& \leq C^{2} c_{\Omega}^{\left[t_{0}-t-2\right]} \inf _{x \in D} \varphi_{G}\left(x, t_{0}\right) \leq C^{2} e^{\left(\log c_{\Omega}\right)\left(t_{0}-t\right)} \inf _{x \in D} \varphi_{G}\left(x, t_{0}\right),
\end{aligned}
$$

where $x_{\Omega}, c_{\Omega}$ are as in (5.1), $C>0$ depends only on $D, d$, and the structure of $L$, and $[t]$ denotes the integer part of a real number $t$.

Our goal is to show now that, roughly speaking, the inequality (5.4) is preserved if the supremum on the left hand side is taken over all of $G$. For that purpose let $g$ be as in Lemma 3.4 with $a_{0}, b_{0}, \delta=\delta_{0}$ as chosen in the paragraph preceding Remark 5.1 and with $K=\bar{G} \backslash D_{0}$ (note that by smoothness of $G$ one has $|K|=$ $\left.\left|G \backslash D_{0}\right| \leq\left|\Omega \backslash D_{0}\right|<\delta_{0}\right)$. Denote $c_{2}=C^{2} \inf _{x \in D} \varphi_{G}\left(x, t_{0}\right)$. Define the following function:

$$
v_{G}(x, t)=\frac{\left(c_{2}-e^{-\left(\log c_{\Omega}\right)\left(t_{0}-t\right)} \varphi_{G}(x, t)\right)}{g(x)}+\frac{c_{2}}{2} \quad\left(x \in \bar{G} \backslash D, t \leq t_{0}-2\right) .
$$


We will show that this function is nonnegative on $(\bar{G} \backslash D) \times\left(-\infty, t_{0}-2\right]$. First, we have

$$
L\left(v_{G} g\right)=g\left(a_{i j} D_{i j} v_{G}+\hat{b}_{i} D_{i} v_{G}+\hat{c}_{0} v_{G}\right),
$$

where

$$
\begin{array}{r}
\hat{b}_{i}=\left(a_{i j}+a_{j i}\right) D_{j} g / g+b_{i}, \\
\hat{c}_{0}=\left(a_{i j} D_{i j} g+b_{i} D_{i} g\right) / g+c_{0}=L g / g,
\end{array}
$$

and, by Lemma 3.4 and our choice of $a_{0}, b_{0}$ above,

$$
\hat{c}_{0}=L g / g \leq\left(\sqrt{N} \beta_{0}-b_{0}\right)(|\nabla g|+g) / g \leq \sqrt{N} \beta_{0}-b_{0} \leq-2 \beta_{0}-3 \log c_{\Omega} .
$$

Next,

$$
\begin{aligned}
\left(v_{G}\right)_{t} g & =-\left(\log c_{\Omega}\right) e^{-\left(\log c_{\Omega}\right)\left(t_{0}-t\right)} \varphi_{G}-e^{-\left(\log c_{\Omega}\right)\left(t_{0}-t\right)}\left(\varphi_{G}\right)_{t} \\
& =\left(\log c_{\Omega}\right)\left(v_{G} g-\frac{c_{2}}{2} g-c_{2}\right)+L\left(-e^{-\left(\log c_{\Omega}\right)\left(t_{0}-t\right)} \varphi_{G}\right) \\
& =\left(\log c_{\Omega}\right)\left(v_{G} g-\frac{c_{2}}{2} g-c_{2}\right)+L\left(v_{G} g-\frac{c_{2}}{2} g-c_{2}\right) .
\end{aligned}
$$

Using the above relations and the fact that $1 \leq g \leq 2$ we get

$$
\begin{aligned}
\left(v_{G}\right)_{t} & =a_{i j} D_{i j} v_{G}+\hat{b}_{i} D_{i} v_{G}+\left(\hat{c}_{0}+\log c_{\Omega}\right) v_{G} \\
& +c_{2}\left(-\frac{1}{2} \log c_{\Omega}-\frac{1}{g} \log c_{\Omega}-\frac{1}{2} \hat{c}_{0}-\frac{c_{0}}{g}\right) \\
& \geq a_{i j} D_{i j} v_{G}+\hat{b}_{i} D_{i} v_{G}+\left(\hat{c}_{0}+\log c_{\Omega}\right) v_{G},
\end{aligned}
$$

where $\hat{c}_{0}+\log c_{\Omega} \leq-2 \beta_{0}-2 \log c_{\Omega} \leq-2 \beta_{0}$ and the inequality holds on $(G \backslash \bar{D}) \times$ $\left(-\infty, t_{0}-2\right)$. Note that (5.4) and the definition of $c_{2}$ imply

$$
v_{G} \geq 0 \quad \text { on } \bar{D} \times\left(-\infty, t_{0}-2\right] .
$$

Hence, since $\varphi_{G}=0$ on $\partial G \times \mathbb{R}, v_{G} \geq 0$ on $\partial(G \backslash \bar{D}) \times\left(-\infty, t_{0}-2\right]$. The maximum principle then implies

$$
\left\|v_{G}^{-}(\cdot, t)\right\|_{L^{\infty}(G \backslash \bar{D})} \leq e^{-2 \beta_{0}(t-\tau)}\left\|v_{G}^{-}(\cdot, \tau)\right\|_{L^{\infty}(G \backslash \bar{D})} \quad\left(t_{0}-2 \geq t \geq \tau\right) .
$$

By the definition of $v_{G}$ and Theorem 3.8 one has $\left(\tau \leq t_{0}-2\right)$

$$
\begin{aligned}
\left\|v_{G}^{-}(\cdot, \tau)\right\|_{L^{\infty}(G \backslash \bar{D})} & \leq e^{-\left(\log c_{\Omega}\right)\left(t_{0}-\tau\right)}\left\|\varphi_{G}(\cdot, \tau)\right\|_{L^{\infty}(G \backslash \bar{D})} \\
& \leq \frac{e^{-\left(\log c_{\Omega}\right)\left(t_{0}-\tau\right)}}{C_{G}(D)} \sup _{x \in D} \varphi_{G}(x, \tau),
\end{aligned}
$$

where $C_{G}(D)$ is as in (3.14). Applying (5.4) with $t=\tau$ to the last inequality above and using the definition of $c_{2}$, we obtain for any $\tau \leq t_{0}-2$,

$$
\left\|v_{G}^{-}(\cdot, \tau)\right\|_{L^{\infty}(G \backslash \bar{D})} \leq \frac{c_{2}}{C_{G}(D)}
$$

Sending $\tau$ to $-\infty$ in (5.7) and taking (5.8) into account, we get that $v_{G}^{-} \equiv 0$ on $(G \backslash \bar{D}) \times\left(-\infty, t_{0}-2\right]$ and hence (using also (5.6) $) v_{G} \geq 0$ on $G \times\left(-\infty, t_{0}-2\right]$. Elementary inequalities then give $\left\|\varphi_{G}\left(\cdot, t_{0}-2\right)\right\|_{L^{\infty}(G)} \leq 2 c_{2} e^{2 \log c_{\Omega}}$. Using finally the maximum principle and the definition of $c_{2}$ we derive

$$
\begin{aligned}
\left\|\varphi_{G}\left(\cdot, t_{0}\right)\right\|_{L^{\infty}(G)} & \leq e^{2 \beta_{0}}\left\|\varphi_{G}\left(\cdot, t_{0}-2\right)\right\|_{L^{\infty}(G)} \leq 2 c_{2} e^{2\left(\beta_{0}+\log c_{\Omega}\right)} \\
& =2 C^{2} e^{2\left(\beta_{0}+\log c_{\Omega}\right)} \inf _{x \in D} \varphi_{G}\left(x, t_{0}\right),
\end{aligned}
$$


which implies (5.3) with $C_{1}=(1 / 2) C^{-2} e^{-2\left(\beta_{0}+\log c_{\Omega}\right)}$. In view of the dependences of $c_{\Omega}$ and $C$, the constant $C_{1}$ depends only on $\operatorname{inrad}(\Omega), D, d$, and the structure of $L$. The proof is complete.

As a simple consequence we obtain the following corollary.

Corollary 5.3. Let $D_{0}, D, G, d, \varphi_{G}$ be as in Lemma [5.2. Then

$$
e^{\beta_{0}} \geq \frac{\left\|\varphi_{G}(t+\tau)\right\|_{L^{\infty}(G)}}{\left\|\varphi_{G}(t)\right\|_{L^{\infty}(G)}} \geq C_{2}(D, d) \quad(t \in \mathbb{R}, \tau \in[0,1])
$$

and the constant $C_{2}(D, d)>0$ depends only on $\operatorname{inrad}(\Omega), D, d$, and the structure of $L$.

Proof. The inequality on the left follows from the maximum principle (Proposition 3.2). By the Harnack inequality and Lemma 5.2 we have for any $x \in D$,

$$
C \varphi_{G}(x, t+\tau) \geq \varphi_{G}(x, t+\tau-1) \geq C_{1}(D, d)\left\|\varphi_{G}(\cdot, t+\tau-1)\right\|_{L^{\infty}(G)},
$$

where $C>0$ depends only on $D, d$, and the structure of $L$. Again using the maximum principle, we get $\left\|\varphi_{G}(\cdot, t)\right\|_{L^{\infty}(G)} \leq e^{\beta_{0}}\left\|\varphi_{G}(\cdot, t+\tau-1)\right\|_{L^{\infty}(G)}$ for $\tau \in$ $[0,1]$, which in combination with the above inequalities implies the right hand side inequality in (5.9) with $C_{2}(D, d)=C^{-1} C_{1}(D, d) e^{-\beta_{0}}$.

We next state a lemma, which will enable us to prove that $\varphi_{\Omega}$ to be defined below is a solution of (1.1) on $\Omega \times \mathbb{R}$.

Lemma 5.4. Let $D_{0}, D, G, d, \varphi_{G}$ be as in Lemma 5.2 and $w_{G}$ as in Proposition 4.3. Then

$$
\frac{\varphi_{G}(x, t)}{\left\|\varphi_{G}(\cdot, t)\right\|_{L^{\infty}(G)}} \leq C_{3}(D, d) w_{G}(x, t) \quad((x, t) \in G \times \mathbb{R}),
$$

where $C_{3}=2\left(\beta_{0}-\log C_{2}\right) / C_{2}$ and $C_{2}$ is as in Corollary [5.3.

Proof. Fix $t_{0} \in \mathbb{R}$. Note that Corollary 5.3 implies that

$$
\frac{\left\|\varphi_{G}(t)\right\|_{L^{\infty}(G)}}{\left\|\varphi_{G}\left(t_{0}\right)\right\|_{L^{\infty}(G)}} \leq\left(1 / C_{2}\right) e^{\log \left(1 / C_{2}\right)\left(t_{0}-t\right)} \quad\left(t \leq t_{0}\right)
$$

and we may assume without loss of generality that $C_{2}<1$. We set

$$
v(x, t)=w_{G}(x, t)-\frac{C_{2} e^{\log \left(1 / C_{2}\right)\left(t-t_{0}\right)}}{2\left(\beta_{0}-\log C_{2}\right)} \frac{\varphi_{G}(x, t)}{\left\|\varphi_{G}\left(t_{0}\right)\right\|_{L^{\infty}(G)}} \quad\left(x \in G, t \leq t_{0}\right) .
$$

Then by the bound on $w_{G}$ (see (4.11) ) and (5.11), the function $v$ is bounded and $v=0$ on $\partial G \times\left(-\infty, t_{0}\right]$. Moreover, an easy computation shows that for some positive $\epsilon$ we have

$$
v_{t}-M v \geq \frac{1}{2} \geq-\epsilon v \quad \text { on } \quad G \times\left(-\infty, t_{0}\right) .
$$

By the maximum principle we get

$$
\left\|v^{-}\left(\cdot, t_{0}\right)\right\|_{L^{\infty}(G)} \leq e^{-\epsilon\left(t_{0}-\tau\right)}\left\|v^{-}(\cdot, \tau)\right\|_{L^{\infty}(G)} \quad\left(t_{0} \geq \tau\right) .
$$

Sending $\tau \rightarrow-\infty$ and using the boundedness of $v$ we obtain $v^{-}\left(\cdot, t_{0}\right) \equiv 0$ on $G$. Using this fact, we obtain (5.10) as claimed.

We are ready to state the following existence theorem, which in particular yields Theorem 2.1 (cf. [3, Theorem 2.1]). 
Theorem 5.5. There exists a positive entire solution $\varphi_{\Omega}$ of (1.1) such that the following holds. If $D$ is a subdomain of $\Omega$ with $\bar{D} \subset \Omega$, then

$$
C_{4}(D) \leq \frac{\varphi_{\Omega}(x, t)}{\left\|\varphi_{\Omega}(\cdot, t)\right\|_{L^{\infty}(\Omega)}} \leq C_{5} w_{\Omega}(x, t) \quad((x, t) \in D \times \mathbb{R}) .
$$

The constant $C_{4}(D)>0$ depends only on $D, \Omega$ and the structure of $L$. The constant $C_{5}$ depends only on $\Omega$ and the structure of $L$. Moreover, $\varphi_{\Omega}$ satisfies the estimate (5.9) (with " $G=\Omega$ ") with a constant $C_{2}>0$ depending only on $\Omega$ and the structure of $L$.

Proof. Let $\Omega_{j} \subset \subset \Omega_{j+1} \subset \subset \Omega$ be a sequence of smooth subdomains such that $\bigcup_{j=1}^{\infty} \Omega_{j}=\Omega$, and for each $j$ let $\varphi_{\Omega_{j}}$ be the positive entire solution given by Theorem 3.8 and satisfying $\left\|\varphi_{\Omega_{j}}(\cdot, 0)\right\|_{L^{\infty}\left(\Omega_{j}\right)}=1$. The above results along with standard parabolic regularity guarantee that, at least for a subsequence, the functions $\varphi_{\Omega_{j}}$ converge locally uniformly on $\Omega \times \mathbb{R}$ to a positive entire solution $\varphi_{\Omega}$ of (1.1), which in addition satisfies the bounds (5.13), (5.9). The proof is complete.

\section{Stabilizing Properties of Positive solutions}

For $D_{0}$ as in (5.2) denote

$$
d_{0}=\operatorname{dist}\left(\bar{D}_{0}, \partial \Omega\right)>0 .
$$

We start with the following theorem.

Theorem 6.1. Let $D_{0}$ be as in (5.2). Then there exists a domain $D_{1}$ with $D_{0} \subset \subset$ $D_{1} \subset \subset \Omega$ and such that the following holds. Let $D, G$ be domains with $G$ smooth and such that $D_{1} \subseteq D \subset \subset G \subseteq \Omega$ and $\operatorname{dist}(\bar{D}, \partial G) \geq d>0$. Let $v$ be a positive solution of (3.1), (3.2) on $G \times(-1, \infty)$. Then there exists a constant $C_{6}(D, d)>0$ and $t_{0}(v) \in\left[0,2+\log _{2}\left(\|v(0)\|_{L^{\infty}(G)} /\left(\inf _{x \in D} v(x, 0)\right)\right)\right]$ such that for any $t \geq t_{0}(v)$ we have

$$
\frac{v(x, t)}{\|v(\cdot, t)\|_{L^{\infty}(G)}} \geq C_{6}(D, d) \quad(x \in D) .
$$

The constant $C_{6}(D, d)$ depends only on $\operatorname{inrad}(\Omega), D_{0}, d_{0}, D, d$ and the structure of $L$.

Remark 6.2. For a similar result for divergence form parabolic equations we refer the reader to [13, Lemma 5.1]. A stronger version of the above estimate is contained in [17, Lemma 3.9] under the assumption that $\Omega$ is Lipschitz.

We prove Theorem 6.1 via two lemmas, which will also be useful in the proof of Theorem 2.2. Before we state them let us specify the domain $D_{1}$ in Theorem 6.1 Denote

$$
\tilde{C}_{2}=C_{2}\left(D_{0}, d_{0} / 8\right)
$$

where $d_{0}$ is as in (6.1) and $C_{2}\left(D_{0}, d_{0} / 8\right)$ is as in Corollary 5.3. Next, with $c_{\Omega}$ as in (5.1), let $k>0$ be so large that $2 e^{-k}<\left(\tilde{C}_{2} / 16 c_{\Omega}\right)$ and let $\delta>0$ be the corresponding number given by Lemma 3.5. We now choose a smooth domain $D_{1}$ with $D_{0} \subset \subset D_{1} \subset \subset \Omega$ such that $\operatorname{dist}\left(\bar{D}_{0}, \partial D_{1}\right) \geq d_{0} / 8$ and $\left|\Omega \backslash D_{1}\right|<\delta$. For future use we also note that, with $x_{\Omega}$ as in (5.1), $B\left(x_{\Omega}, \operatorname{inrad}(\Omega) / 2\right) \subset D_{1}$, which follows from (5.2) and $D_{0} \subset D_{1}$. Thus, setting $\rho=\operatorname{inrad}(\Omega) / 4$ in Theorem 3.6 and making $D_{1}$ larger if necessary, we may assume $\left|\Omega \backslash D_{1}\right|<\delta$, where $\delta=$ 
$\delta\left(N, \operatorname{diam}(\Omega), \alpha_{0}, \beta_{0}, \operatorname{inrad}(\Omega) / 4\right)$ is as in Theorem 3.6. This is the domain $D_{1}$ that will serve our purpose.

For $x_{\Omega}, c_{\Omega}$ as above and $v, D, d$ as in Theorem 6.1 define the function

$$
\eta(t)=v\left(x_{\Omega}, t\right) /\|v(\cdot, t)\|_{L^{\infty}(G)} \quad(t>-1),
$$

and set

$$
\tilde{C}(D, d)=\frac{C_{1}(D, d)\left(\tilde{C}_{2}\right)^{2}}{16 c_{\Omega} e^{2 \beta_{0}}},
$$

where $C_{1}(D, d)$ is as in Lemma 5.2 and $\tilde{C}_{2}$ is as in (6.3). Our first lemma in this section states that if a positive solution of (3.1), (3.2) is concentrating near the boundary of $G$ over a unit of time, then its norm drops significantly during that time interval.

Lemma 6.3. Let $v, d, D, G$ be as in Theorem 6.1, let $\tilde{C}(D, d), \tilde{C}_{2}, c_{\Omega}$, and $\eta$ be as in (6.5) and (6.4), respectively. Further, for $k>0$ let $\delta$ be as in Lemma 3.5 and suppose $|G \backslash \bar{D}|<\delta$. Suppose for some $t_{0} \geq 0$ and $\alpha \in(0,1]$ we have

$$
\frac{\|v(t)\|_{L^{\infty}(D)}}{\|v(t)\|_{L^{\infty}(G)}} \leq \alpha \tilde{C}(D, d) \quad\left(t \in\left[t_{0}, t_{0}+1\right]\right) .
$$

Then

$$
\frac{\left\|v\left(t_{0}+1\right)\right\|_{L^{\infty}(G)}}{\left\|v\left(t_{0}\right)\right\|_{L^{\infty}(G)}} \leq \min \left\{\frac{\alpha \tilde{C}_{2}}{16 c_{\Omega}}+2 e^{-k}, \frac{\left\|\varphi_{G}\left(t_{0}+1\right)\right\|_{L^{\infty}(G)}}{2\left\|\varphi_{G}\left(t_{0}\right)\right\|_{L^{\infty}(G)}}\right\},
$$

and

$$
\eta\left(t_{0}+1\right)>2 \eta\left(t_{0}\right) .
$$

Proof. We start with the proof of the first inequality in (6.7). Define the function

$$
u(x, t)=\left(\frac{\alpha \tilde{C}_{2}}{16 c_{\Omega} e^{\beta_{0}}} \frac{\left\|v\left(t_{0}\right)\right\|_{L^{\infty}(G)}}{\left\|\varphi_{G}\left(t_{0}\right)\right\|_{L^{\infty}(G)}}\right) \varphi_{G}(x, t)-v(x, t) \quad\left((x, t) \in G \times\left[t_{0}, \infty\right)\right) .
$$

We are going to prove now that $u(x, t) \geq 0$ for all $x \in \partial D, t \in\left[t_{0}, t_{0}+1\right]$. First, by the assumption (6.6) and by the maximum principle, we have $\|v(t)\|_{L^{\infty}(D)} \leq$ $\tilde{C}(D, d)\|v(t)\|_{L^{\infty}(G)} \leq \tilde{C}(D, d) e^{\beta_{0}}\left\|v\left(t_{0}\right)\right\|_{L^{\infty}(G)}$ for $t \in\left[t_{0}, t_{0}+1\right]$. Next, using Lemma 5.2 we get $\varphi_{G}(x, t) \geq C_{1}(D, d)\left\|\varphi_{G}(t)\right\|_{L^{\infty}(G)}$ for all $x \in D$ and by Corollary 5.3 (with $D=D_{0}$ and $d=d_{0} / 8$ ) we obtain $\left\|\varphi_{G}(t)\right\|_{L^{\infty}(G)} \geq \tilde{C}_{2}\left\|\varphi_{G}\left(t_{0}\right)\right\|_{L^{\infty}(G)}$ for all $t \in\left[t_{0}, t_{0}+1\right]$ and $\tilde{C}_{2}$ as in (6.3). Combining these inequalities, one easily concludes that $u(x, t) \geq 0$ for all $x \in \partial D, t \in\left[t_{0}, t_{0}+1\right]$. Thus $u$ is a supersolution of (3.1), (3.2) with $G$ replaced by $G \backslash \bar{D}$ and since $|G \backslash \bar{D}|<\delta$ we can use Lemma 3.5 with the corresponding $k$. Therefore

$$
\left\|u^{-}\left(t_{0}+1\right)\right\|_{L^{\infty}(G \backslash \bar{D})} \leq 2 e^{-k}\left\|u^{-}\left(t_{0}\right)\right\|_{L^{\infty}(G \backslash \bar{D})} \leq 2 e^{-k}\left\|v\left(t_{0}\right)\right\|_{L^{\infty}(G)} .
$$

Now observe that an elementary estimate and the maximum principle give

$$
\begin{aligned}
\left\|u^{-}\left(t_{0}+1\right)\right\|_{L^{\infty}(G \backslash \bar{D})} & \geq\left\|v\left(t_{0}+1\right)\right\|_{L^{\infty}(G \backslash \bar{D})} \\
& -\left(\frac{\alpha \tilde{C}_{2}}{16 c_{\Omega} e^{\beta_{0}}} \frac{\left\|v\left(t_{0}\right)\right\|_{L^{\infty}(G)}}{\left\|\varphi_{G}\left(t_{0}\right)\right\|_{L^{\infty}(G)}}\right)\left\|\varphi_{G}\left(t_{0}+1\right)\right\|_{L^{\infty}(G)} \\
& \geq\left\|v\left(t_{0}+1\right)\right\|_{L^{\infty}(G \backslash \bar{D})}-\frac{\alpha \tilde{C}_{2}}{16 c_{\Omega}}\left\|v\left(t_{0}\right)\right\|_{L^{\infty}(G)} .
\end{aligned}
$$


Since $\alpha \leq 1$ and we may assume $\tilde{C}(D, d)<1$, (6.6) with $t=t_{0}+1$ implies $\left\|v\left(t_{0}+1\right)\right\|_{L^{\infty}(G \backslash \bar{D})}=\left\|v\left(t_{0}+1\right)\right\|_{L^{\infty}(G)}$. Using this fact and the above estimates we arrive at

$$
\left\|v\left(t_{0}+1\right)\right\|_{L^{\infty}(G)} \leq\left(\frac{\alpha \tilde{C}_{2}}{16 c_{\Omega}}+2 e^{-k}\right)\left\|v\left(t_{0}\right)\right\|_{L^{\infty}(G)},
$$

which gives the first inequality in (6.7).

To prove the remaining inequality in (6.7), note that now we may assume $2 e^{-k}<$ $\tilde{C}_{2} /\left(16 c_{\Omega}\right)$. Indeed, this follows from our choice of $D_{1}$ in the paragraph following Remark 6.2 and from the fact that $|G \backslash \bar{D}| \leq\left|\Omega \backslash \bar{D}_{1}\right|$. Thus, by Corollary 5.3 (with $D=D_{0}$ and $\left.d=d_{0} / 8\right)$, we also get

$$
\frac{\alpha \tilde{C}_{2}}{16 c_{\Omega}}+2 e^{-k} \leq \frac{\tilde{C}_{2}}{8 c_{\Omega}} \leq \frac{1}{8} \frac{\left\|\varphi_{G}\left(t_{0}+1\right)\right\|_{L^{\infty}(G)}}{\left\|\varphi_{G}\left(t_{0}\right)\right\|_{L^{\infty}(G)}}
$$

which together with (6.9) implies the second inequality in (6.7).

To prove (6.8), we first use the Harnack inequality to obtain

$$
v\left(x_{\Omega}, t_{0}\right) \leq c_{\Omega} v\left(x_{\Omega}, t_{0}+1\right) .
$$

This inequality in combination with (6.9) and the left inequality in (6.10) yields $\eta\left(t_{0}+1\right) \geq\left(8 / \tilde{C}_{2}\right) \eta\left(t_{0}\right)$. Since we may assume that $\tilde{C}_{2}<1$ (see (6.3) and Corollary [5.3), (6.8) follows. The proof is thus complete.

Lemma 6.4. Let $v, d, D, G$ be as in Theorem 6.1 and suppose that for some $\kappa>0, t_{0} \geq 0$ we have

$$
\frac{\left\|v\left(t_{0}\right)\right\|_{L^{\infty}(D)}}{\left\|v\left(t_{0}\right)\right\|_{L^{\infty}(G)}} \geq \kappa
$$

Then there exists a constant $C_{7}(D, d)>0$ such that

$$
\frac{v(x, t)}{\|v(t)\|_{L^{\infty}(G)}} \geq C_{7}(D, d) \kappa \quad\left(x \in D, t \geq t_{0}+1\right) .
$$

The constant $C_{7}(D, d)$ depends only on $\operatorname{inrad}(\Omega), D_{0}, d_{0}, D, d$, and the structure of $L$.

Proof. Fix $\kappa \in(0,1]$ as in the statement of Lemma 6.4. By the Harnack inequality and the maximum principle we have the following implication for any $t \geq 0$ and any $\tilde{\kappa} \in(0,1]$ :

$$
\frac{\|v(t)\|_{L^{\infty}(D)}}{\|v(t)\|_{L^{\infty}(G)}} \geq \tilde{\kappa} \Rightarrow \eta(t+\tau) \geq C \tilde{\kappa} \quad(\tau \in[1,3])
$$

where $C>0$ depends only on $D, d$, and the structure of $L$ and $\eta$ is as in (6.4). By Lemma 6.3 we also have for any $t \geq 0$ :

$$
\frac{\|v(\tau)\|_{L^{\infty}(D)}}{\|v(\tau)\|_{L^{\infty}(G)}} \leq \tilde{C}(D, d) \text { for } \tau \in[t, t+1] \Rightarrow \eta(t+1)>2 \eta(t) .
$$

For $C, \tilde{C}(D, d)$ as above we define the open set

$$
A=\left\{t>t_{0}+1: \eta(t)<C \tilde{C}(D, d) \kappa\right\}
$$

Suppose the interval $(a, b)$ is a connected component of $A$. The assumption (6.11) inserted into the implication (6.13) with $t=t_{0}$ and $\tilde{\kappa}=\kappa$ gives that $\eta\left(t_{0}+1\right) \geq$ $C \kappa>C \tilde{C}(D, d) \kappa$ (because $\tilde{C}(D, d)<1)$. Therefore $a>t_{0}+1$ and by continuity 
of $\eta$ we have $\eta(a)=C \tilde{C}(D, d) \kappa$. Now, the definition of $A$, the contrapositive of (6.13) with $\tilde{\kappa}=\tilde{C}(D, d) \kappa$ and (6.14) imply that if $l \leq b-a-1$ is a positive integer, then

$$
C \tilde{C}(D, d) \kappa>\eta(a+l)>2^{l} \eta(a)=2^{l} C \tilde{C}(D, d) \kappa,
$$

which is impossible. Therefore necessarily $b-a<2$. This shows that for each $t \geq t_{0}+1$ there exists $r \in[t, t+2]$ such that $\eta(r) \geq C \tilde{C}(D, d) \kappa$. Then by the Harnack inequality and the maximum principle, $\inf _{x \in D} v(x, s) /\|v(s)\|_{L^{\infty}(G)} \geq C_{7} \kappa$ for $s \in[t+3, t+6]$ and some $C_{7}>0$ depending only on $\operatorname{inrad}(\Omega), D_{0}, d_{0}, D, d$, and the structure of $L$. Hence (6.12) holds for any $t \geq t_{0}+4$, and for $t \in\left[t_{0}+1, t_{0}+4\right]$, it follows from (6.11), the Harnack inequality and the maximum principle.

Proof of Theorem 6.1. First of all, we have $\eta(0) \geq \inf _{x \in D} v(x, 0) /\|v(0)\|_{L^{\infty}(G)}$. Lemma 6.3 with $\alpha=1$ and the fact that $\eta \leq 1$ then guarantee that there exists a smallest

$$
\tau_{0} \in\left[0,1+\log _{2}\left(\|v(0)\|_{L^{\infty}(G)} /\left(\inf _{x \in D} v(x, 0)\right)\right)\right]
$$

for which

$$
\frac{\left\|v\left(\tau_{0}\right)\right\|_{L^{\infty}(D)}}{\left\|v\left(\tau_{0}\right)\right\|_{L^{\infty}(G)}} \geq \tilde{C}(D, d)
$$

where $\tilde{C}(D, d)$ is as in (6.5). The inequality (6.2) then follows from Lemma 6.4 with $\kappa=\tilde{C}(D, d)$, and we can take $t_{0}(v)=\tau_{0}+1$.

We will need the following corollary in the sequel.

Corollary 6.5. Let $v, d, D, G$ be as in Theorem 6.1 and suppose that for some $\kappa>0, t_{0} \geq 0$ we have

$$
\frac{\left\|v\left(t_{0}\right)\right\|_{L^{\infty}(D)}}{\left\|v\left(t_{0}\right)\right\|_{L^{\infty}(G)}} \geq \kappa
$$

Then

$$
\frac{\|v(t)\|_{L^{\infty}(G)}}{\|v(s)\|_{L^{\infty}(G)}} \geq \kappa C_{8}(D, d) e^{-\left(\log c_{\Omega}\right)(t-s)} \quad\left(t \geq s \geq t_{0}+1\right),
$$

where $c_{\Omega}$ is as in (5.1) and the constant $C_{8}(D, d)>0$ depends only on $\operatorname{inrad}(\Omega)$, $D_{0}, d_{0}, D, d$, and the structure of $L$.

Proof. By the Harnack inequality and by our assumption on $v$ we obtain

$$
C_{H} v\left(x, t_{0}+\tau\right) \geq\left\|v\left(t_{0}\right)\right\|_{L^{\infty}(D)} \geq \kappa\left\|v\left(t_{0}\right)\right\|_{L^{\infty}(G)} \quad(x \in D, \tau \in[1,3]),
$$

where $C_{H}>0$ depends only on $D, d$, and the structure of $L$. The maximum principle gives $\left\|v\left(t_{0}+\tau\right)\right\|_{L^{\infty}(G)} \leq e^{3 \beta_{0}}\left\|v\left(t_{0}\right)\right\|_{L^{\infty}(G)}$ for all $\tau \in[1,3]$. The latter two sets of inequalities imply the following estimate:

$$
\frac{\left\|v\left(t_{0}+\tau_{2}\right)\right\|_{L^{\infty}(G)}}{\left\|v\left(t_{0}+\tau_{1}\right)\right\|_{L^{\infty}(G)}} \geq \frac{\kappa}{C_{H} e^{3 \beta_{0}}} \quad\left(1 \leq \tau_{1} \leq \tau_{2} \leq 3\right) .
$$


To finish the proof observe that if $t \geq s \geq t_{0}+3$, then by the Harnack inequality, (6.12) and the maximum principle,

$$
\begin{aligned}
\|v(t)\|_{L^{\infty}(G)} & \geq v\left(x_{\Omega}, t\right) \geq \frac{1}{c_{\Omega}} v\left(x_{\Omega}, t-1\right) \\
& \geq \cdots \geq \frac{1}{c_{\Omega}^{[t-s]+1}} v\left(x_{\Omega}, t-[t-s]-1\right) \\
& \geq \frac{C_{7}(D, d) \kappa}{c_{\Omega}^{[t-s]+1}}\|v(t-[t-s]-1)\|_{L^{\infty}(G)} \\
& \geq \frac{C_{7}(D, d) \kappa}{e^{\beta_{0}} c_{\Omega}^{t-s+1}}\|v(s)\|_{L^{\infty}(G)},
\end{aligned}
$$

where $x_{\Omega}$ and $c_{\Omega}$ are as in (5.1) and [t] denotes the integer part of a real number $t$. The above inequalities imply (6.17).

The following lemma compares the growth of a positive solution of (3.1), (3.2) with the growth of any other solution.

Lemma 6.6. Let $v, d, D, G$ be as in Theorem 6.1 and for $t_{0} \geq 0$ let $u$ be a solution of (3.1), (3.2) on $G \times\left(t_{0}, \infty\right)$ with $u\left(\cdot, t_{0}\right) \in C_{0}(\bar{G})$. There exists a constant $C_{9}(D, d) \in(0,1)$ depending only on $\operatorname{inrad}(\Omega), \operatorname{diam}(\Omega), D_{0}, d_{0}, D, d$, and the structure of $L$ such that the following holds. Suppose that for some $\kappa>0$ we have

$$
\left\|v\left(t_{0}\right)\right\|_{L^{\infty}(D)} \geq \kappa\left\|v\left(t_{0}\right)\right\|_{L^{\infty}(G)} \geq C_{9}^{-1}(D, d)\left\|u\left(t_{0}\right)\right\|_{L^{\infty}(G)} .
$$

Then

$$
v(x, t)>|u(x, t)| \quad\left(x \in \bar{D}, t \geq t_{0}+1\right)
$$

and

$$
\|v(t)\|_{L^{\infty}(G)} \geq(\kappa / 3)\|u(t)\|_{L^{\infty}(G)} \quad\left(t \geq t_{0}+1\right) .
$$

Proof. For $u, v, t_{0}$ as in the lemma define the function

$$
\tilde{u}(x, t)=v(x, t)-u(x, t) \quad\left((x, t) \in G \times\left[t_{0}+1, \infty\right)\right) .
$$

To prove (6.18) we are going to apply Theorem 3.6 to $\tilde{u}$ with $\rho=\operatorname{inrad}(\Omega) / 4$, $\theta=1 / 8, d=\operatorname{dist}(\bar{D}, \partial G) / 2$, and $\tau=t_{0}+1$. Note that our choice of $D_{1}$ above (see the paragraph immediately following Remark 6.2) and the fact that $D_{1} \subseteq D$ imply that the assumptions (3.10) and (3.11) in Theorem 3.6 are satisfied. We need to verify the remaining assumptions in this theorem.

By positivity of $v$ and the maximum principle we have

$$
\left\|\tilde{u}^{-}\left(t_{0}+r\right)\right\|_{L^{\infty}(G)} \leq\left\|u\left(t_{0}+r\right)\right\|_{L^{\infty}(G)} \leq e^{2 \beta_{0}}\left\|u\left(t_{0}\right)\right\|_{L^{\infty}(G)} \quad(r \in[1,2]) .
$$

By the Harnack inequality and the assumption on $u, v$ we have

$$
\begin{aligned}
\left\|v\left(t_{0}+r\right)\right\|_{L^{\infty}(G)} & \geq v\left(x, t_{0}+r\right) \geq C_{H}\left\|v\left(t_{0}\right)\right\|_{L^{\infty}(D)} \\
& \geq C_{H} C_{9}^{-1}(D, d)\left\|u\left(t_{0}\right)\right\|_{L^{\infty}(G)} \quad(x \in D, r \in[1,2]),
\end{aligned}
$$


where $C_{H}>0$ depends only on $D, d$ and the structure of $L$. Hence for all $x \in \bar{D}$ one has

$$
\begin{aligned}
\|\tilde{u}\|_{L^{\infty}\left(D \times\left(t_{0}+\frac{9}{8}, t_{0}+\frac{10}{8}\right)\right)} & \geq \tilde{u}\left(x, t_{0}+\frac{9}{8}\right) \geq v\left(x, t_{0}+\frac{9}{8}\right)-e^{2 \beta_{0}}\left\|u\left(t_{0}\right)\right\|_{L^{\infty}(G)} \\
& \geq\left(C_{H} C_{9}^{-1}(D, d)-e^{2 \beta_{0}}\right)\left\|u\left(t_{0}\right)\right\|_{L^{\infty}(G)} \\
& =\mu^{-1} e^{2 \beta_{0}}\left\|u\left(t_{0}\right)\right\|_{L^{\infty}(G)},
\end{aligned}
$$

where $\mu$ is as in Theorem 3.6 and $C_{9}(D, d)=\mu(\mu+1)^{-1} C_{H} e^{-2 \beta_{0}}$ depends only on $\operatorname{inrad}(\Omega), \operatorname{diam}(\Omega), D, d$, and the structure of $L$. The inequality (6.21) with $r=1$ and (6.23) show that (3.13) in Theorem 3.6 is satisfied. Finally, we note that if we replace $t_{0}+\frac{9}{8}$ in (6.23) by any $t \in\left[t_{0}+1, t_{0}+2\right]$, then all of these inequalities, except for the first one, remain valid. This gives that $\tilde{u}$ is positive on $\bar{D} \times\left[t_{0}+1, t_{0}+2\right]$ and thus also the assumption (3.12) in Theorem 3.6 is satisfied. Therefore, by $(s 1)$ in Theorem 3.6, $v(x, t)>u(x, t)$ for all $(x, t) \in \bar{D} \times\left[t_{0}+1, \infty\right)$. The same arguments as above apply if we define $\tilde{u}=v+u$, showing that also $v(x, t)>-u(x, t)$ for all $(x, t) \in \bar{D} \times\left[t_{0}+1, \infty\right)$. The proof of (6.18) is finished.

Using (6.17) with $s=t_{0}+1$ and (6.22) with $r=1$ we obtain

$$
\|v(t)\|_{L^{\infty}(G)} \geq \frac{\kappa C_{8}(D, d) C_{H}}{C_{9}(D, d)} e^{-\left(\log c_{\Omega}\right)\left(t-t_{0}-1\right)}\left\|u\left(t_{0}\right)\right\|_{L^{\infty}(G)} \quad\left(t \geq t_{0}+1\right) .
$$

Since we have already proved (6.18) we may apply Lemma 3.5 to $\tilde{u}=v \pm u$ with $U=G \backslash \bar{D}$ and $k$ such that $k>\log c_{\Omega}+\log 32$. The inequality for $k$ follows from the fact that $D_{1} \subseteq D$ and our choice of $D_{1}$ (see the paragraph following Remark 6.2). As a consequence we get for any $t \geq t_{0}+1$,

$$
\begin{aligned}
\left\|(v(t) \pm u(t))^{-}\right\|_{L^{\infty}(G \backslash \bar{D})} & \leq 2 e^{-k\left(t-t_{0}-1\right)}\left\|\left(v\left(t_{0}+1\right) \pm u\left(t_{0}+1\right)\right)^{-}\right\|_{L^{\infty}(G \backslash \bar{D})} \\
& \leq 2 e^{-k\left(t-t_{0}-1\right)}\left\|u\left(t_{0}+1\right)\right\|_{L^{\infty}(G)} \\
& \leq 2 e^{\beta_{0}} e^{-k\left(t-t_{0}-1\right)}\left\|u\left(t_{0}\right)\right\|_{L^{\infty}(G) .}
\end{aligned}
$$

Using $k>\log c_{\Omega}+\log 32$, (6.24) and (6.25), making $C_{9}(D, d)$ smaller if necessary, we obtain

$$
\left\|(v(t) \pm u(t))^{-}\right\|_{L^{\infty}(G \backslash \bar{D})} \leq(1 / \kappa)\|v(t)\|_{L^{\infty}(G)} \quad\left(t \geq t_{0}+1\right) .
$$

Clearly,

$$
\pm u(x, t)-v(x, t) \leq\left\|(v(t) \mp u(t))^{-}\right\|_{L^{\infty}(G \backslash \bar{D})} \quad\left(x \in G \backslash \bar{D}, t \geq t_{0}+1\right) .
$$

The last two inequalities combined imply

$$
\|u(t)\|_{L^{\infty}(G \backslash \bar{D})} \leq(1+1 / \kappa)\|v(t)\|_{L^{\infty}(G)} \quad\left(t \geq t_{0}+1\right) .
$$

Therefore, using also (6.18), we derive

$$
\begin{aligned}
\|u(t)\|_{L^{\infty}(G)} & \leq\|u(t)\|_{L^{\infty}(D)}+\|u(t)\|_{L^{\infty}(G \backslash \bar{D})} \\
& \leq\|v(t)\|_{L^{\infty}(G)}+(1+1 / \kappa)\|v(t)\|_{L^{\infty}(G)} \\
& =(2+1 / \kappa)\|v(t)\|_{L^{\infty}(G) \quad\left(t \geq t_{0}+1\right),}
\end{aligned}
$$

which implies (6.19) since $\kappa \in(0,1]$. 
The next lemma compares the growth of any positive solution $v$ of (3.1), (3.2) with the growth of $\varphi_{G}$.

Lemma 6.7. Let v, $d, D, G, D_{1}$ be as in Theorem 6.1 and assume $D=D_{1}$. Let $\tau_{0}$ be as in (6.15) with $D=D_{1}$. Then there exist constants $C_{10}\left(D_{1}, d\right), C_{11}\left(D_{1}, d\right)>1$ such that the following inequalities hold:

$$
\begin{aligned}
\frac{\|v(t)\|_{L^{\infty}(G)}}{\left\|\varphi_{G}(t)\right\|_{L^{\infty}(G)}} & \leq C_{10}\left(D_{1}, d\right) \frac{\|v(0)\|_{L^{\infty}(G)}}{\left\|\varphi_{G}(0)\right\|_{L^{\infty}(G)}} \quad(t \geq 0), \\
\quad\left(C_{11}\right)^{-1} & \leq \frac{\|v(t)\|_{L^{\infty}(G)}}{\left\|\varphi_{G}(t)\right\|_{L^{\infty}(G)}} \frac{\left\|\varphi_{G}(s)\right\|_{L^{\infty}(G)}}{\|v(s)\|_{L^{\infty}(G)}} \leq C_{11} \quad\left(t \geq s \geq \tau_{0}+1\right) .
\end{aligned}
$$

These constants depend only on $\operatorname{inrad}(\Omega), \operatorname{diam}(\Omega), D_{0}, d_{0}, D_{1}, d$, and the structure of $L$.

Remark 6.8. Heuristically, as soon as $v\left(x, \tau_{0}\right)$ becomes comparable with its norm $\left\|v\left(\tau_{0}\right)\right\|_{L^{\infty}(G)}$ for some $x \in D_{1}$ and $\tau_{0} \geq 0$, then the norms $\|v(t)\|_{L^{\infty}(G)}$ and $\left\|\varphi_{G}(t)\right\|_{L^{\infty}(G)}$ will grow at the same rate for all $t \geq \tau_{0}+1$.

Proof of Lemma 6.7. Let $\tau_{0} \geq 0$ be as defined in (6.15) with $D=D_{1}$. Let $t \in$ $\left[0, \tau_{0}+1\right]$. Then by the maximum principle and Corollary 5.3 we have

$$
\frac{\|v(t)\|_{L^{\infty}(G)}}{\left\|\varphi_{G}(t)\right\|_{L^{\infty}(G)}} \leq \frac{e^{2 \beta_{0}}}{\tilde{C}_{2}^{2}} \frac{\|v([t-1])\|_{L^{\infty}(G)}}{\left\|\varphi_{G}([t-1])\right\|_{L^{\infty}(G)}}
$$

where $\tilde{C}_{2}$ is as in (6.3), $[t-1]$ denotes the integer part of $t-1$ and we make a temporary convention that $[t-1]=0$ if $t \in[0,1]$. Note that, by the definition of $\tau_{0}$ and since $[t-1] \leq\left[\tau_{0}\right]$, the inequality (6.6) (with $D=D_{1}$ and $\alpha=1$ ) is satisfied for all $t \in\left[0,\left[\tau_{0}\right]\right]$. We may therefore use Lemma 6.3 (with $D=D_{1}$ ) to obtain (still respecting our temporary convention)

$$
\frac{\|v([t-1])\|_{L^{\infty}(G)}}{\left\|\varphi_{G}([t-1])\right\|_{L^{\infty}(G)}} \leq \frac{1}{2^{[t-1]}} \frac{\|v(0)\|_{L^{\infty}(G)}}{\left\|\varphi_{G}(0)\right\|_{L^{\infty}(G)}} .
$$

Clearly, the above inequalities show that (6.26) holds for all $t \in\left[0, \tau_{0}+1\right]$.

Let us prove (6.26) in the remaining case, i.e., for $t \geq \tau_{0}+1$. The choice of $\tau_{0}$ above implies that (6.16) holds with $D=D_{1}$, i.e., we have

$$
\frac{\left\|v\left(\tau_{0}\right)\right\|_{L^{\infty}\left(D_{1}\right)}}{\left\|v\left(\tau_{0}\right)\right\|_{L^{\infty}(G)}} \geq \tilde{C}\left(D_{1}, d\right)
$$

By Lemma 5.2 also

$$
\frac{\left\|\varphi_{G}\left(\tau_{0}\right)\right\|_{L^{\infty}\left(D_{1}\right)}}{\left\|\varphi_{G}\left(\tau_{0}\right)\right\|_{L^{\infty}(G)}} \geq C_{1}\left(D_{1}, d\right)
$$

We are going to apply Lemma 6.6 with $\kappa=\min \left\{\tilde{C}\left(D_{1}, d\right), C_{1}\left(D_{1}, d\right)\right\}, t_{0}=\tau_{0}$, and with

$$
v(x, t)=\frac{\varphi_{G}(x, t)}{\kappa C_{9}\left(D_{1}, d\right)\left\|\varphi_{G}\left(\tau_{0}\right)\right\|_{L^{\infty}(G)}}, u(x, t)=\frac{v(x, t)}{\left\|v\left(\tau_{0}\right)\right\|_{L^{\infty}(G)}} .
$$

To avoid confusion, we remark that the $v$ in the definition of $u$ in (6.30) is as in the statement of Lemma 6.7 and has nothing to do with the $v$ appearing on the left in 
(6.30). Applying Lemma 6.6. we obtain

$$
\frac{\left\|\varphi_{G}(t)\right\|_{L^{\infty}(G)}}{\kappa C_{9}\left(D_{1}, d\right)\left\|\varphi_{G}\left(\tau_{0}\right)\right\|_{L^{\infty}(G)}} \geq \frac{\kappa\|v(t)\|_{L^{\infty}(G)}}{3\left\|v\left(\tau_{0}\right)\right\|_{L^{\infty}(G)}} \quad\left(t \geq \tau_{0}+1\right),
$$

which finishes the proof of (6.26).

Finally, note that we may interchange the roles of $\varphi_{G}$ and $v$ in (6.30). We then get (6.31) with the roles of $\varphi_{G}$ and $v$ interchanged. These two versions of (6.31) yield (6.27).

For future use we state a corollary of Lemma 6.7.

Corollary 6.9. Let $d, D, G, D_{1}$ be as in Theorem 6.1 and assume $D=D_{1}$. Let $u$ be any solution of (3.4) with $u_{0} \in C_{0}(\bar{G})$. Then

$$
\frac{\|u(t)\|_{L^{\infty}(G)}}{\left\|\varphi_{G}(t)\right\|_{L^{\infty}(G)}} \leq \tilde{C}_{10}\left(D_{1}, d\right) \frac{\left\|u_{0}\right\|_{L^{\infty}(G)}}{\left\|\varphi_{G}(0)\right\|_{L^{\infty}(G)}} \quad(t \geq 0)
$$

where $\tilde{C}_{10}\left(D_{1}, d\right)=e^{\beta_{0}} C_{10}\left(D_{1}, d\right) / C_{2}\left(D_{1}, d\right)$ and $C_{2}$ and $C_{10}$ are as in Corollary 5.3 (with $D=D_{1}$ ) and Lemma 6.7, respectively.

Proof. By uniqueness of the solutions of (3.4) we can write

$$
u(x, t)=u_{1}\left(x, t ; 0, u_{0}^{+}\right)-u_{2}\left(x, t ; 0, u_{0}^{-}\right)
$$

for all $x \in G$ and $t \geq 0$. Here, $u_{1}\left(x, t ; 0, u_{0}^{+}\right)$is the (nonnegative) solution of (3.4) with $u_{0}=u_{0}^{+}$( $u_{2}$ is defined analogously). By the maximum principle $\|u(t)\|_{L^{\infty}(G)} \leq$ $e^{\beta_{0}}\left\|u_{0}\right\|_{L^{\infty}(G)}$ for all $t \in[0,1]$ and by Corollary 5.3 we have $\left\|\varphi_{G}(t)\right\|_{L^{\infty}(G)} \geq$ $C_{2}\left(D_{1}, d\right)\left\|\varphi_{G}(0)\right\|_{L^{\infty}(G)}$. Hence the inequality (6.32) holds for $t \in[0,1]$ and with $\tilde{C}_{10}$ replaced by $e^{\beta_{0}} / C_{2}\left(D_{1}, d\right)$. If $t>1$ we have by Lemma 6.7 and by what we have just said above,

$$
\begin{aligned}
\frac{\|u(t)\|_{L^{\infty}(G)}}{\left\|\varphi_{G}(t)\right\|_{L^{\infty}(G)}} & \leq \frac{\max \left\{\left\|u_{1}(t)\right\|_{L^{\infty}(G)},\left\|u_{2}(t)\right\|_{L^{\infty}(G)}\right\}}{\left\|\varphi_{G}(t)\right\|_{L^{\infty}(G)}(G)} \\
& \leq C_{10}\left(D_{1}, d\right) \frac{\max \left\{\left\|u_{1}(1)\right\|_{L^{\infty}(G)},\left\|u_{2}(1)\right\|_{L^{\infty}(G)}\right\}}{\left\|\varphi_{G}(1)\right\|_{L^{\infty}(G)}} \\
& \leq \frac{e^{\beta_{0}} C_{10}\left(D_{1}, d\right)}{C_{2}\left(D_{1}, d\right)} \frac{\max \left\{\left\|u_{0}^{+}\right\|_{L^{\infty}(G)},\left\|u_{0}^{-}\right\|_{L^{\infty}(G)}\right\}}{\left\|\varphi_{G}(0)\right\|_{L^{\infty}(G)}} \\
& =\frac{e^{\beta_{0}} C_{10}\left(D_{1}, d\right)}{C_{2}\left(D_{1}, d\right)} \frac{\left\|u_{0}\right\|_{L^{\infty}(G)}}{\left\|\varphi_{G}(0)\right\|_{L^{\infty}(G)}} .
\end{aligned}
$$

Proposition 4.13 gives the following corollary.

Corollary 6.10. Theorem 6.1, Lemmas 6.3 6.7, and Corollaries 6.5 and 6.9 remain valid if $G$ is replaced by $\Omega$, i.e., if $v$ is a positive solution of (1.1) on $\Omega \times(-1, \infty)$ and if $u$ is a solution of (1.1) or an associated initial value problem (4.16). All of these results hold in the corresponding forms on arbitrary intervals $(s, \infty), s \in \mathbb{R}$.

We conclude this section with a result which shows "eventual" positivity of all solutions of (3.1), (3.2), which stay positive on a sufficiently large subdomain of a smooth domain $G$. 
Proposition 6.11. Let $D_{1}$ and $G$ be as in Theorem 6.1 and let $u$ be a solution of (3.1), (3.2) on $G \times(s, \infty)$ such that $u(x, t)>0$ for all $x \in D_{1}$ and $t \geq t_{0}>s$. Then there exists $t_{G}(u)>t_{0}$ such that $u(x, t)>0$ for all $x \in G$ and $t \geq t_{G}(u)$.

Remark 6.12. The proof of Proposition 6.11 shows that it is sufficient to assume that $G$ is a Lipschitz (bounded) domain. We point out, however, that if $G$ is not smooth enough, then Proposition 6.11 need not hold. In [13] we have constructed an example of a bounded domain $\Omega$ satisfying a (uniform) exterior cone condition at each point of $\partial \Omega$ and such that if $L=\triangle$ in (1.1), then there exists a solution $u$ of (1.1) on $\Omega \times(0, \infty)$ with the following properties. For all $t>0$ we have $u(x(t), t)=0$ for some $x(t) \in \Omega$, and for any subdomain $D \subset \subset \Omega$ there exists $t_{D}>0$ such that $u(x, t)>0$ for all $x \in D$ and $t \geq t_{D}$.

Proof of Proposition 6.11. By Lemma 3.5 (with " $U=G \backslash \bar{D}_{1}$ ") we have

$$
\left\|u^{-}(t)\right\|_{L^{\infty}\left(G \backslash \bar{D}_{1}\right)} \leq 2 e^{-k\left(t-t_{0}-1\right)}\left\|u^{-}\left(t_{0}+1\right)\right\|_{L^{\infty}\left(G \backslash \bar{D}_{1}\right)} \quad\left(t \geq t_{0}+1\right)
$$

where $k$ is chosen in the paragraph following Remark 6.2. On the other hand, with $x_{\Omega}$ and $c_{\Omega}$ as in (5.1), we have by the Harnack inequality,

$$
u\left(x_{\Omega}, t\right) \geq e^{-\left(\log c_{\Omega}\right)\left(t-t_{0}-1\right)} u\left(x_{\Omega}, t_{0}+1\right) \quad\left(t \geq t_{0}+2\right) .
$$

Since $k>\log c_{\Omega}$, the above inequalities imply that there exists $\tilde{t} \geq t_{0}+2$ such that

$$
u\left(x_{\Omega}, \tilde{t}+1\right)>e^{\beta_{0}} C_{G}\left\|u^{-}(\tilde{t})\right\|_{L^{\infty}\left(G \backslash \bar{D}_{1}\right)},
$$

where $C_{G}$ is as in Theorem 3.7 (with $\delta_{0}=1$ in that theorem). By uniqueness of the solutions of (3.4) we have

$$
u(x, t)=u_{1}\left(x, t ; \tilde{t}, u^{+}(\tilde{t})\right)-u_{2}\left(x, t ; \tilde{t}, u^{-}(\tilde{t})\right)
$$

for all $x \in G$ and $t \geq \tilde{t}$. Clearly

$$
\begin{array}{ll}
u^{+}(x, t) \leq u_{1}\left(x, t ; \tilde{t}, u^{+}(\tilde{t})\right) & (x \in G, t \geq \tilde{t}), \\
u^{-}(x, t) \leq u_{2}\left(x, t ; \tilde{t}, u^{-}(\tilde{t})\right) & (x \in G, t \geq \tilde{t}) .
\end{array}
$$

If $u^{-}(\cdot, \tilde{t}) \equiv 0$ on $\Omega$, then the conclusion follows. We may therefore assume $u^{-}(\cdot, \tilde{t}) \not \equiv$ 0 and proceed as follows. Theorem 3.7 applied to $u_{1}$ and $u_{2}$ gives

$$
C_{G} \inf _{x \in G} \frac{u_{1}(x, \tilde{t}+1)}{u_{2}(x, \tilde{t}+1)} \geq \sup _{x \in G} \frac{u_{1}(x, \tilde{t}+1)}{u_{2}(x, \tilde{t}+1)} .
$$

Since $x_{\Omega} \in D_{1}$, the positivity assumption on $u$ in $D_{1}$ implies $u^{+}\left(x_{\Omega}, \tilde{t}+1\right)=$ $u\left(x_{\Omega}, \tilde{t}+1\right)$. Then, by (6.37), the supremum on the right may be estimated from below by

$$
\frac{u\left(x_{\Omega}, \tilde{t}+1\right)}{\left\|u_{2}(\tilde{t}+1)\right\|_{L^{\infty}(G)}} \geq \frac{u\left(x_{\Omega}, \tilde{t}+1\right)}{e^{\beta_{0}}\left\|u^{-}(\tilde{t})\right\|_{L^{\infty}(G)}}=\frac{u\left(x_{\Omega}, \tilde{t}+1\right)}{e^{\beta_{0}}\left\|u^{-}(\tilde{t})\right\|_{L^{\infty}\left(G \backslash \bar{D}_{1}\right)}}>C_{G},
$$

where we have also used the maximum principle, positivity of $u(\cdot, \tilde{t})$ in $D_{1}$, and (6.35). The above inequalities imply $u_{1}(x, \tilde{t}+1)>u_{2}(x, \tilde{t}+1)$ for all $x \in G$, which in view of (6.36) means that $u(x, \tilde{t}+1)>0$ for all $x \in G$. By the maximum principle and the Harnack inequality we then get $u(x, t)>0$ for all $x \in G$ and $t \geq t_{G}(u)$, where $t_{G}(u)=\tilde{t}+1$. We conclude by noting that for the above proof to work it suffices to assume that $G$ is a Lipschitz domain. This follows from Proposition 3.2 and Theorem 3.7 


\section{Some EStimates OF SIGN-CHANGING SOLUTIONS}

We feel that it may be helpful for the reader if we give some heuristic arguments justifying the lemmas to follow. To establish the estimate (2.3) we use the following strategy. For a smooth domain $G$ (sufficiently close to $\Omega$ ) and a given time-interval we analyze the relative decrease of $u$, a sign-changing solution of (3.4), with respect to $\varphi_{G}$ during that time-interval. More precisely, if $\|u(t+5)\|_{L^{\infty}(G)} /\|u(t)\|_{L^{\infty}(G)} \ll$ $\left\|\varphi_{G}(t+5)\right\|_{L^{\infty}(G)} /\left\|\varphi_{G}(t)\right\|_{L^{\infty}(G)}$, then we simply move on from $t$ to $t+5$. If, on the other hand, $\|u(t+\tau)\|_{L^{\infty}(G)} /\|u(t)\|_{L^{\infty}(G)} \approx\left\|\varphi_{G}(t+\tau)\right\|_{L^{\infty}(G)} /\left\|\varphi_{G}(t)\right\|_{L^{\infty}(G)}$ for $\tau \in[0,5]$ (up to a certain multiple), that is, if $u$ does not drop "too much" compared to $\varphi_{G}$, then Lemma 7.1 below guarantees that the solutions $u\left(\cdot, \tilde{t} ; t, u^{+}(t)\right)$, $u\left(\cdot, \tilde{t} ; t, u^{-}(t)\right)$ are comparable to each other away from the boundary of $G$ for all $\tilde{t} \geq t+T_{0}$ and some $T_{0} \geq 1$ independent of $u_{0}$ and $G$. Lemma 7.2 then guarantees that there is enough "cancellation" between the positive and negative parts of $u$ to result in dropping of the norm of $u$ with respect to $\varphi_{G}$.

Denote

$$
d_{1}=\operatorname{dist}\left(\bar{D}_{1}, \partial \Omega\right)>0,
$$

where $D_{1}$ is as in Section 6 , Let

$$
\tilde{C}_{10}=\tilde{C}_{10}\left(D_{1}, d_{1} / 8\right),
$$

where $\tilde{C}_{10}\left(D_{1}, d_{1} / 8\right)$ is as in Corollary 6.9. Let $k>0$ be so large that (with $\tilde{C}_{2}$ as in (6.3))

$$
2 e^{-k}<\left(\tilde{C}_{2}\right)^{5} /\left(4 e^{4 \beta_{0}}\left(\tilde{C}_{10}\right)^{2}\right)
$$

and let $\delta>0$ be the corresponding number given by Lemma 3.5. Let us fix a smooth domain $D_{2}$ with

$$
D_{1} \subset \subset D_{2} \subset \subset \Omega,
$$

and such that $\left|\Omega \backslash D_{2}\right|<\delta$ and $\operatorname{dist}\left(\bar{D}_{1}, \partial D_{2}\right) \geq d_{1} / 8$. In the following lemma we use that for any solution $u$ of (3.4) we may write

$$
u(x, t)=u_{1}\left(x, t ; 0, u_{0}^{+}\right)-u_{2}\left(x, t ; 0, u_{0}^{-}\right) \quad((x, t) \in G \times[0, \infty)) .
$$

Lemma 7.1. Let $G$ be a smooth domain such that $D_{2} \subset \subset G \subseteq \Omega$ and $\operatorname{dist}\left(\bar{D}_{2}, \partial G\right)$ $\geq d>0$. Let $u$ be a solution of (3.4) with $u_{0} \in C_{0}(\bar{G})$ and such that $u(\cdot, t)$ vanishes somewhere in $G$ for all $t>0$. Suppose that

$$
\frac{\|u(\tau)\|_{L^{\infty}(G)}}{\left\|u_{0}\right\|_{L^{\infty}(G)}} \geq \frac{\left(\tilde{C}_{2}\right)^{5}}{2\left(\tilde{C}_{10}\right)^{2}} \quad(\tau \in[0,5])
$$

where $\tilde{C}_{2}$ and $\tilde{C}_{10}$ are as in (6.3) and (7.2), respectively. Then, with $u_{1}$ and $u_{2}$ as in (7.5), there exist constants $C_{12} \in(0,1)$ and $T_{0}>1$ with the following properties. We have

$$
\frac{u_{i}(x, t)}{\left\|u_{j}(t)\right\|_{L^{\infty}(G)}} \geq C_{12} \quad\left(x \in D_{2}, t \geq T_{0}, i, j=1,2\right) .
$$

The constants $C_{12}$ and $T_{0}$ depend only on $\operatorname{inrad}(\Omega), \operatorname{diam}(\Omega), D_{0}, d_{0}, D_{1}, d_{1}, D_{2}$, $d$, and the structure of $L$. 
Proof. Since $\|u(\tau)\|_{L^{\infty}(G)} \leq \max \left\{\left\|u_{1}(\tau)\right\|_{L^{\infty}(G)},\left\|u_{2}(\tau)\right\|_{L^{\infty}(G)}\right\}$ for all $\tau \geq 0$, (7.6) with $\tau=5$ implies that we may assume, say,

$$
\left\|u_{1}(5)\right\|_{L^{\infty}(G)} \geq \frac{\left(\tilde{C}_{2}\right)^{5}}{2\left(\tilde{C}_{10}\right)^{2}}\left\|u_{0}\right\|_{L^{\infty}(G)} .
$$

We claim that for some $t \in[1,2]$ we must have

$$
\frac{\left\|u_{1}(t)\right\|_{L^{\infty}\left(D_{2}\right)}}{\left\|u_{1}(t)\right\|_{L^{\infty}(G)}}>\frac{C_{1}\left(D_{2}, d\right)\left(\tilde{C}_{2}\right)^{6}}{16 c_{\Omega} e^{6 \beta_{0}}\left(\tilde{C}_{10}\right)^{2}}
$$

where $C_{1}\left(D_{2}, d\right)$ is as in Lemma 5.2, $\tilde{C}_{2}, \tilde{C}_{10}$ are as above and $c_{\Omega}$ is as in (5.1). Indeed, suppose (7.9) is not true. This contradiction assumption allows us to use Lemma 6.3 with $D=D_{2}, \alpha=\left(\tilde{C}_{2}\right)^{4} / e^{4 \beta_{0}}\left(\tilde{C}_{10}\right)^{2}$ and $k$ as in (7.3). Using that lemma and (2.3) we get

$$
\begin{aligned}
& \left\|u_{1}(5)\right\|_{L^{\infty}(G)} \leq e^{3 \beta_{0}}\left\|u_{1}(2)\right\|_{L^{\infty}(G)} \leq e^{3 \beta_{0}}\left(\frac{\alpha \tilde{C}_{2}}{16 c_{\Omega}}+2 e^{-k}\right)\left\|u_{1}(1)\right\|_{L^{\infty}(G)} \\
& <e^{3 \beta_{0}}\left(\frac{\left(\tilde{C}_{2}\right)^{5}}{16 c_{\Omega} e^{4 \beta_{0}\left(\tilde{C}_{10}\right)^{2}}}+\frac{\left(\tilde{C}_{2}\right)^{5}}{4 e^{4 \beta_{0}}\left(\tilde{C}_{10}\right)^{2}}\right)\left\|u_{1}(1)\right\|_{L^{\infty}(G)} \\
& \leq \frac{\left(\tilde{C}_{2}\right)^{5}}{2 e^{\beta_{0}}\left(\tilde{C}_{10}\right)^{2}}\left\|u_{1}(1)\right\|_{L^{\infty}(G)} \leq \frac{\left(\tilde{C}_{2}\right)^{5}}{2\left(\tilde{C}_{10}\right)^{2}}\left\|u_{0}^{+}\right\|_{L^{\infty}(G)} \\
& \leq \frac{\left(\tilde{C}_{2}\right)^{5}}{2\left(\tilde{C}_{10}\right)^{2}}\left\|u_{0}\right\|_{L^{\infty}(G)},
\end{aligned}
$$

contradicting (7.8). Thus (7.9) holds and we note that the constant on the right hand side of that inequality depends only on $\operatorname{inrad}(\Omega), \operatorname{diam}(\Omega), D_{0}, d_{0}, D_{1}, d_{1}$, $D_{2}, d$ and the structure of $L$. Lemma 6.4 with $D=D_{2}$ and $\kappa$ equal to the right hand side in (7.9) then gives

$$
\frac{u_{1}(x, t)}{\left\|u_{1}(t)\right\|_{L^{\infty}(G)}} \geq C \quad\left(x \in D_{2}, t \geq 3\right)
$$

and $C>0$ depends only on $\operatorname{inrad}(\Omega), \operatorname{diam}(\Omega), D_{0}, d_{0}, D_{1}, d_{1}, D_{2}, d$ and the structure of $L$.

Our next goal is to prove that a similar estimate holds for $u_{2}$ and that the norms $\left\|u_{1}(t)\right\|_{L^{\infty}(G)}$ and $\left\|u_{2}(t)\right\|_{L^{\infty}(G)}$ are comparable (in a suitable sense) for all $t \geq T_{0}$ and some $T_{0}>1$ with the required dependence. With $C$ as in (17.10), suppose that the assumptions of Lemma 6.6 are satisfied for $D=D_{2}, v=u_{1}, u=u_{2}, \kappa=C$ and some $t_{0} \geq 3$. We would then have $u(x, t)=u_{1}(x, t)-u_{2}(x, t)>0$ for all $x \in \bar{D}_{2}$ and $t \geq t_{0}+1$. Then Proposition 6.11 would imply $u(x, \tilde{t})>0$ for all $x \in G$ and $\tilde{t} \geq t_{G}(u)$, contradicting the assumption that $u(\cdot, t)$ vanishes somewhere in $G$ for all $t>0$. Thus, necessarily

$$
\left\|u_{2}(t)\right\|_{L^{\infty}(G)}>C_{9}\left(D_{2}, d\right) C\left\|u_{1}(t)\right\|_{L^{\infty}(G)} \quad(t \geq 3),
$$

where $C_{9}\left(D_{2}, d\right)$ is as in Lemma 6.6

To motivate the estimates below we note that if we knew that (7.10) holds with $u_{1}$ replaced by $u_{2}$ and all $t \geq s_{0}$ for an appropriate $s_{0}$, then we would get (7.11) for all $t \geq s_{0}$ with the roles of $u_{1}$ and $u_{2}$ interchanged, which in combination with (7.10) (valid for both $u_{1}$ and $u_{2}$ ) would imply the conclusion of the lemma. On the other hand, if $u_{2}(\cdot, t)$ were very small in $D_{2}$ compared to $\left\|u_{2}(t)\right\|_{L^{\infty}(G)}$ on a long 
time-interval, then by Lemma 6.3 the norm $\left\|u_{2}(t)\right\|_{L^{\infty}(G)}$ would be decaying very fast, eventually leading to a contradiction with (7.11).

We claim

$$
\left(C_{11}\right)^{-1} \leq \frac{\left\|u_{1}(s)\right\|_{L^{\infty}(G)}}{\left\|\varphi_{G}(s)\right\|_{L^{\infty}(G)}} \frac{\left\|\varphi_{G}(t)\right\|_{L^{\infty}(G)}}{\left\|u_{1}(t)\right\|_{L^{\infty}(G)}} \leq C_{11} \quad(t \geq s \geq 4),
$$

where $C_{11}>1$ depends only on $\operatorname{inrad}(\Omega), \operatorname{diam}(\Omega), D_{0}, d_{0}, D_{1}, d_{1}, D_{2}, d$ and the structure of $L$. Indeed, making use of (7.10), this can be proved in exactly the same way as we proved (6.27) (see also Remark 6.8). We are now going to use Lemma 6.3 with $D=D_{2}$. Suppose that for some positive integer $l$ the inequality (6.6) with $\alpha=1$ (and with $D=D_{2}$ ) is satisfied for $u_{2}$ and all $t \in[5,5+l]$. Using Lemma 6.3 $l$ times followed by an application of (7.12) and (7.8), we compute

$$
\begin{aligned}
\left\|u_{2}(5+l)\right\|_{L^{\infty}(G)} & \leq \frac{\left\|u_{2}(5)\right\|_{L^{\infty}(G)}}{2^{l}} \frac{\left\|\varphi_{G}(5+l)\right\|_{L^{\infty}(G)}}{\left\|\varphi_{G}(5)\right\|_{L^{\infty}(G)}} \\
& \leq \frac{e^{5 \beta_{0}}\left\|u_{0}\right\|_{L^{\infty}(G)}}{2^{l}} \frac{C_{11}\left\|u_{1}(5+l)\right\|_{L^{\infty}(G)}}{\left\|u_{1}(5)\right\|_{L^{\infty}(G)}} \\
& \leq \frac{C_{11} e^{5 \beta_{0}} 2\left(\tilde{C}_{10}\right)^{2}\left\|u_{1}(5)\right\|_{L^{\infty}(G)}}{2^{l}\left(\tilde{C}_{2}\right)^{5}} \frac{\left\|u_{1}(5+l)\right\|_{L^{\infty}(G)}}{\left\|u_{1}(5)\right\|_{L^{\infty}(G)}} \\
& =\frac{C_{11} e^{5 \beta_{0}} 2\left(\tilde{C}_{10}\right)^{2}}{2^{l}\left(\tilde{C}_{2}\right)^{5}}\left\|u_{1}(5+l)\right\|_{L^{\infty}(G)} .
\end{aligned}
$$

Comparing this estimate with (7.11), we get an upper bound on $l$, call it $l_{0}$, depending only on $\operatorname{inrad}(\Omega), \operatorname{diam}(\Omega), D_{0}, d_{0}, D_{1}, d_{1}, D_{2}, d$ and the structure of $L$. Therefore, for some $t \in\left[5, l_{0}+6\right]$ the inequality (6.6) (with $\alpha=1$ and $D=D_{2}$ ) is violated. Then by Lemma 6.4 we get

$$
\frac{u_{2}(x, t)}{\left\|u_{2}(t)\right\|_{L^{\infty}(G)}} \geq C \quad\left(x \in D_{2}, t \geq l_{0}+7\right)
$$

and $C>0$ depends only on $\operatorname{inrad}(\Omega), \operatorname{diam}(\Omega), D_{0}, d_{0}, D_{1}, d_{1}, D_{2}, d$ and the structure of $L$.

Having established (7.13), the same proof as above shows that (7.11) holds with the roles of $u_{1}$ and $u_{2}$ interchanged and with $t \geq l_{0}+9$. Set $T_{0}=l_{0}+9$. The inequalities (7.10), (7.13), and (7.11) (for both $u_{1}$ and $u_{2}$ and $t \geq T_{0}$ ) imply that (7.7) holds. The proof is complete.

The following lemma will be crucial in our considerations.

Lemma 7.2. Let $u, G, d, \tilde{C}_{2}, \tilde{C}_{10}$ be as in Lemma 7.1 and suppose that (77.6) holds. There exists a constant $T_{1}>0$, depending only on $\operatorname{inrad}(\Omega), \operatorname{diam}(\Omega), D_{0}$, $d_{0}, D_{1}, d_{1}, D_{2}, d$ and the structure of $L$, with the following property. For any $t \geq 2 T_{1}$ we have

$$
\begin{aligned}
& \left\|u\left(t ; T_{1}, u^{+}\left(T_{1}\right)\right)\right\|_{L^{\infty}(G)} \leq \sigma_{0}\left\|u\left(t ; 0, u^{+}(0)\right)\right\|_{L^{\infty}(G)}, \\
& \left\|u\left(t ; T_{1}, u^{-}\left(T_{1}\right)\right)\right\|_{L^{\infty}(G)} \leq \sigma_{0}\left\|u\left(t ; 0, u^{-}(0)\right)\right\|_{L^{\infty}(G)},
\end{aligned}
$$

where $\sigma_{0}=1-C_{12} / 4$ and $C_{12}$ is as in Lemma 7.1.

Proof. Set

$$
\omega_{1}=1-C_{12} / 2
$$


With $T_{0}$ as in Lemma 7.1 set $\widetilde{T}_{0}=T_{0}+1$ and consider the function

$$
\tilde{u}(x, t)=\omega_{1} u\left(x, t ; 0, u^{+}(0)\right)-u(x, t) \quad\left(x \in G, t \geq \widetilde{T}_{0}\right) .
$$

By uniqueness of the solutions of (3.4) we have

$$
u(x, t)=u\left(x, t ; 0, u^{+}(0)\right)-u\left(x, t ; 0, u^{-}(0)\right) \quad(x \in G, t \geq 0) .
$$

This fact and (7.7) with $i=2, j=1$ imply

$$
\begin{aligned}
\tilde{u}(x, t) & =u\left(x, t ; 0, u^{-}(0)\right)-\left(C_{12} / 2\right) u\left(x, t ; 0, u^{+}(0)\right) \\
& \geq C_{12}\left\|u\left(t ; 0, u^{+}(0)\right)\right\|_{L^{\infty}(G)}-\left(C_{12} / 2\right) u\left(x, t ; 0, u^{+}(0)\right) \\
& \geq\left(C_{12} / 2\right)\left\|u\left(t ; 0, u^{+}(0)\right)\right\|_{L^{\infty}(G)}>0 \quad\left(x \in D_{2}, t \geq \widetilde{T}_{0}\right) .
\end{aligned}
$$

We can therefore use Lemma 3.5 with " $U=G \backslash \bar{D}_{2}$ ". We get

$$
\left\|\tilde{u}^{-}(t)\right\|_{L^{\infty}\left(G \backslash \bar{D}_{2}\right)} \leq 2 e^{-k\left(t-\widetilde{T}_{0}\right)}\left\|\tilde{u}^{-}\left(\widetilde{T}_{0}\right)\right\|_{L^{\infty}\left(G \backslash \bar{D}_{2}\right)} \quad\left(t \geq \widetilde{T}_{0}\right),
$$

where $k$ is as in (7.3) and, with $c_{\Omega}$ as in (5.1), we may assume

$$
e^{-k}<1 /\left(2 c_{\Omega}\right) \text {. }
$$

This follows from the fact that $D_{1} \subset D_{2}$ and from the discussion contained in the paragraph following Remark 6.2. Obviously, by the definition of $\tilde{u}$ and (7.18) we have

$$
\left\|\tilde{u}^{-}\left(\widetilde{T}_{0}\right)\right\|_{L^{\infty}\left(G \backslash \bar{D}_{2}\right)} \leq\left\|u^{+}\left(\widetilde{T}_{0}\right)\right\|_{L^{\infty}(G)} \leq\left\|u\left(\widetilde{T}_{0} ; 0, u^{+}(0)\right)\right\|_{L^{\infty}(G)} .
$$

Next, Lemma 7.1 (with $i=j=1$ ) and Corollary 6.5 (with $s=\widetilde{T}_{0}$ ) imply that for any $t \geq \widetilde{T}_{0}$ one has

$$
\left\|u\left(\widetilde{T}_{0} ; 0, u^{+}(0)\right)\right\|_{L^{\infty}(G)} \leq C e^{\left(\log c_{\Omega}\right)\left(t-\widetilde{T}_{0}\right)}\left\|u\left(t ; 0, u^{+}(0)\right)\right\|_{L^{\infty}(G)},
$$

where $C>0$ depends only on $\operatorname{inrad}(\Omega), \operatorname{diam}(\Omega), D_{0}, d_{0}, D_{1}, d_{1}, D_{2}, d$ and the structure of $L$. On the other hand, the definition of $\tilde{u}$ and (7.19) give that for all $x \in G$ and $t \geq \widetilde{T}_{0}$ one also has

$$
\left\|\left.\tilde{u}^{-}(t)\right|_{L^{\infty}\left(G \backslash \bar{D}_{2}\right)}=\right\| \tilde{u}^{-}(t) \|_{L^{\infty}(G)} \geq u^{+}(x, t)-\omega_{1} u\left(x, t ; 0, u^{+}(0)\right),
$$

which clearly implies the following inequality $\left(t \geq \widetilde{T}_{0}\right)$ :

$$
\left\|u^{+}(t)\right\|_{L^{\infty}(G)} \leq\left\|\tilde{u}^{-}(t)\right\|_{L^{\infty}\left(G \backslash \bar{D}_{2}\right)}+\omega_{1}\left\|u\left(t ; 0, u^{+}(0)\right)\right\|_{L^{\infty}(G)} .
$$

By (7.20), (7.22), (7.23) and the first equality in (7.24) we have $\left(t \geq \widetilde{T}_{0}\right)$

$$
\left\|\tilde{u}^{-}(t)\right\|_{L^{\infty}(G)} \leq 2 C e^{\left(-k+\log c_{\Omega}\right)\left(t-\widetilde{T}_{0}\right)}\left\|u\left(t ; 0, u^{+}(0)\right)\right\|_{L^{\infty}(G)} .
$$

Using (7.25) and (7.26), we obtain $\left(t \geq \widetilde{T}_{0}\right)$

$$
\left\|u^{+}(t)\right\|_{L^{\infty}(G)} \leq\left(\omega_{1}+2 C e^{\left(-k+\log c_{\Omega}\right)\left(t-\widetilde{T}_{0}\right)}\right)\left\|u\left(t ; 0, u^{+}(0)\right)\right\|_{L^{\infty}(G)} .
$$

We now fix $t_{1}>\widetilde{T}_{0}+1$ (to be determined below) and define the function $v$ to be the solution of (3.1), (3.2) on $G \times\left[t_{1}, \infty\right)$ with the nonnegative initial condition given for all $x \in G$ by

$$
v\left(x, t_{1}\right)=\omega_{1} u\left(x, t_{1} ; 0, u^{+}(0)\right)-u^{+}\left(x, t_{1}\right)+\left(\omega_{1} u\left(x, t_{1} ; 0, u^{+}(0)\right)-u^{+}\left(x, t_{1}\right)\right)^{-} .
$$

By the uniqueness of the solutions of the initial value problems we can write

$$
v(x, t)=v_{1}(x, t)+v_{2}(x, t) \quad\left(x \in G, t \geq t_{1}\right),
$$


where $v_{1}(x, t)=\omega_{1} u\left(x, t ; 0, u^{+}(0)\right)-u\left(x, t ; t_{1}, u^{+}\left(t_{1}\right)\right)$ and $v_{2}$ is the (nonnegative) solution of (3.1), (3.2) on $G \times\left[t_{1}, \infty\right)$ with

$$
v_{2}\left(x, t_{1}\right)=\left(\omega_{1} u\left(x, t_{1} ; 0, u^{+}(0)\right)-u^{+}\left(x, t_{1}\right)\right)^{-} \quad(x \in G) .
$$

The inequality (7.24) with $t=t_{1}$ implies $\left\|v_{2}\left(t_{1}\right)\right\|_{L^{\infty}(G)} \leq\left\|\tilde{u}^{-}\left(t_{1}\right)\right\|_{L^{\infty}(G)}$ and by the maximum principle $\left\|v_{2}(t)\right\|_{L^{\infty}(G)} \leq e^{\beta_{0}}\left\|v_{2}\left(t_{1}\right)\right\|_{L^{\infty}(G)}$ for any $t \in\left[t_{1}, t_{1}+1\right]$. These inequalities and (7.26) with $t=t_{1}$ imply that for any $t \in\left[t_{1}, t_{1}+1\right]$ we have

$$
\left\|v_{2}(t)\right\|_{L^{\infty}(G)} \leq 2 C e^{\beta_{0}} e^{\left(-k+\log c_{\Omega}\right)\left(t_{1}-\widetilde{T}_{0}\right)}\left\|u\left(t_{1} ; 0, u^{+}(0)\right)\right\|_{L^{\infty}(G)} .
$$

Define finally the function

$$
\tilde{v}(x, t)=\left(\omega_{1}+C_{12} / 8\right) u\left(x, t ; 0, u^{+}(0)\right)-u\left(x, t ; t_{1}, u^{+}\left(t_{1}\right)\right) \quad\left(x \in G, t \geq t_{1}\right) .
$$

Our next goal is to prove, via Theorem [3.6, that $\tilde{v}>0$ on $D_{2} \times\left[t_{1}, \infty\right)$ if $t_{1}$ is suitably chosen. By uniqueness of the solutions of the initial value problems, using the definitions of $v, v_{1}, v_{2}$ and $\tilde{v}$, we see that for any $x \in G$ and $t \geq t_{1}$ one in fact has

$$
\tilde{v}(x, t)=\left(C_{12} / 8\right) u\left(x, t ; 0, u^{+}(0)\right)+v(x, t)-v_{2}(x, t) .
$$

The nonnegativity of $v$ and (7.30) with $t=t_{1}$ then imply

$$
\left\|\tilde{v}^{-}\left(t_{1}\right)\right\|_{L^{\infty}(G)} \leq\left\|v_{2}\left(t_{1}\right)\right\|_{L^{\infty}(G)} \leq 2 C e^{\beta_{0}} e^{\left(-k+\log c_{\Omega}\right)\left(t_{1}-\widetilde{T}_{0}\right)}\left\|u\left(t_{1} ; 0, u^{+}(0)\right)\right\|_{L^{\infty}(G)} .
$$

Next, using Lemma 7.1 (with $i=j=1$ ) and Corollary 6.5 (with $s=t_{1}$ ), we get (7.34)

$$
\left(C_{12} / 8\right) u\left(x, t ; 0, u^{+}(0)\right) \geq \bar{C}\left\|u\left(t_{1} ; 0, u^{+}(0)\right)\right\|_{L^{\infty}(G)} \quad\left(x \in D_{2}, t \in\left[t_{1}, t_{1}+1\right]\right),
$$

where $\bar{C}>0$ depends only on $\operatorname{inrad}(\Omega), \operatorname{diam}(\Omega), D_{0}, d_{0}, D_{1}, d_{1}, D_{2}, d$ and the structure of $L$. Finally, the nonnegativity of $v$ and the inequalities (7.34) and (7.30) imply that for all $x \in \bar{D}_{2}$ and $t \in\left[t_{1}, t_{1}+1\right]$,

$$
\tilde{v}(x, t) \geq\left(\bar{C}-2 C e^{\beta_{0}} e^{\left(-k+\log c_{\Omega}\right)\left(t_{1}-\widetilde{T}_{0}\right)}\right)\left\|u\left(t_{1} ; 0, u^{+}(0)\right)\right\|_{L^{\infty}(G)} .
$$

Let $\mu=\mu\left(N, \operatorname{diam}(\Omega), \alpha_{0}, \beta_{0}, d / 2,1 / 8, \operatorname{inrad}(\Omega) / 4\right)$ be as in Theorem 3.6. It is now easy to see, using $-k+\log c_{\Omega}<-\log 2$ (see (7.21)), (7.33) and (7.35), that if $t_{1}=T_{1}>\widetilde{T}_{0}+1$ is sufficiently large (depending only on $\operatorname{inrad}(\Omega), \operatorname{diam}(\Omega), D_{0}$, $d_{0}, D_{1}, d_{1}, D_{2}, d$ and the structure of $\left.L\right)$, then the assumptions (3.12) and (3.13) in Theorem 3.6 with $D=D_{2}$ are satisfied. The remaining assumptions (3.10) and (3.11) are also satisfied since $D_{1} \subset D_{2}$ (see the choice of $D_{1}$ in the paragraph following Remark 6.2). Therefore, by Theorem 3.6, $\tilde{v}(x, t)>0$ for all $x \in \bar{D}_{2}$ and $t \geq T_{1}$.

The positivity of $\tilde{v}$ on $\bar{D}_{2} \times\left[T_{1}, \infty\right)$ allows us to use similar arguments as those leading to the inequality (7.27). We will only point out the necessary modifications. In the text (and all formulas) appearing between (and including) (7.20) and (7.27) one has to replace everywhere $\tilde{u}$ by $\tilde{v}, \widetilde{T}_{0}$ by $T_{1}$, and $\omega_{1}$ by $\omega_{1}+C_{12} / 8$. With such a replacement, the inequalities (7.20), (7.22) and (7.23) remain valid. In (7.24) we in addition need to replace $u^{+}(x, t)$ by $u\left(x, t ; T_{1}, u^{+}\left(T_{1}\right)\right)$ and in (7.25) and (7.27) the function $u^{+}(t)$ is now replaced by $u\left(t ; T_{1}, u^{+}\left(T_{1}\right)\right)$. With these modifications in place, the inequalities (7.20)-(7.27) continue to hold. Using in particular the 
(modified) inequality (7.27), we get for any $t \geq T_{1}$,

$$
\begin{aligned}
& \left\|u\left(t ; T_{1}, u^{+}\left(T_{1}\right)\right)\right\|_{L^{\infty}(G)} \\
& \leq\left(\omega_{1}+C_{12} / 8+2 C e^{\left(-k+\log c_{\Omega}\right)\left(t-T_{1}\right)}\right)\left\|u\left(t ; 0, u^{+}(0)\right)\right\|_{L^{\infty}(G) .}
\end{aligned}
$$

Obviously, making $T_{1}$ so large that $2 C e^{\left(-k+\log c_{\Omega}\right) T_{1}}<C_{12} / 8$, the inequality (7.36) implies that for any $t \geq 2 T_{1}$ we have

$$
\begin{aligned}
& \left\|u\left(t ; T_{1}, u^{+}\left(T_{1}\right)\right)\right\|_{L^{\infty}(G)} \\
& \leq\left(\omega_{1}+C_{12} / 8+C_{12} / 8\right)\left\|u\left(t ; 0, u^{+}(0)\right)\right\|_{L^{\infty}(G)}=\sigma_{0}\left\|u\left(t ; 0, u^{+}(0)\right)\right\|_{L^{\infty}(G)},
\end{aligned}
$$

as claimed in (7.14). The proof of (7.15) is completely analogous.

Next, we prove the following lemma.

Lemma 7.3. Let $G, d$ be as in Lemma 7.1 and let $u$ be a solution of (3.4) with $u_{0} \in C_{0}(\bar{G})$ and such that $u(\cdot, t)$ vanishes somewhere in $G$ for all $t>0$. There exists a constant $T_{2}>0$, depending only on $\operatorname{inrad}(\Omega)$, $\operatorname{diam}(\Omega), D_{0}, d_{0}, D_{1}, d_{1}$, $D_{2}, d$ and the structure of $L$, such that we have

$$
\frac{\left\|u\left(T_{2}\right)\right\|_{L^{\infty}(G)}}{\left\|\varphi_{G}\left(T_{2}\right)\right\|_{L^{\infty}(G)}} \leq \frac{1}{2} \frac{\left\|u_{0}\right\|_{L^{\infty}(G)}}{\left\|\varphi_{G}(0)\right\|_{L^{\infty}(G)}} .
$$

Proof. Let $m$ be an integer so large that

$$
\sigma_{0}^{m-1}<\frac{1}{2 \tilde{C}_{10}},
$$

where $\sigma_{0}$ is as in Lemma 7.2 and $\tilde{C}_{10}$ is as in (7.2). In view of the dependences of $\sigma_{0}$ and $\tilde{C}_{10}, m$ can be chosen depending only on $\operatorname{inrad}(\Omega), \operatorname{diam}(\Omega), D_{0}, d_{0}, D_{1}$, $d_{1}, D_{2}, d$ and the structure of $L$. We will show that the conclusion of Lemma 7.3 holds with

$$
T_{2}=m T_{1}+5,
$$

where $T_{1}$ is as in Lemma 7.2. We may assume in the remainder of this proof that $u(\cdot, t) \not \equiv 0$ for all $t \in\left[0, T_{2}\right]$ (for otherwise (17.37) is trivially satisfied). We then have the following (mutually exclusive) possibilities:

$$
\frac{\|u(t+\tau)\|_{L^{\infty}(G)}}{\|u(t)\|_{L^{\infty}(G)}} \geq \frac{\left(\tilde{C}_{2}\right)^{5}}{2\left(\tilde{C}_{10}\right)^{2}} \quad\left(\tau \in[0,5], t \in\left[0, m T_{1}\right]\right)
$$

or

(ii) for some $t_{0} \in\left[0, m T_{1}\right]$ and some $\tau_{0} \in[0,5]$,

$$
\frac{\left\|u\left(t_{0}+\tau_{0}\right)\right\|_{L^{\infty}(G)}}{\left\|u\left(t_{0}\right)\right\|_{L^{\infty}(G)}}<\frac{\left(\tilde{C}_{2}\right)^{5}}{2\left(\tilde{C}_{10}\right)^{2}},
$$

where $\tilde{C}_{2}$ and $\tilde{C}_{10}$ are as in (6.3) and (7.2), respectively.

Let us first consider the possibility (i). Writing

$$
u(x, t)=u\left(x, t ;(m-1) T_{1}, u^{+}\left((m-1) T_{1}\right)\right)-u\left(x, t ;(m-1) T_{1}, u^{-}\left((m-1) T_{1}\right)\right)
$$

for $(x, t) \in G \times\left[(m-1) T_{1}, \infty\right)$, we see that

$$
\left\|u^{+}\left(T_{2}\right)\right\|_{L^{\infty}(G)} \leq\left\|u\left(T_{2} ;(m-1) T_{1}, u^{+}\left((m-1) T_{1}\right)\right)\right\|_{L^{\infty}(G)} .
$$


The assumption (i) allows us to use (7.14) $m-1$ times. We get

$$
\begin{aligned}
\| u\left(T_{2} ;(m-1) T_{1},\right. & \left.u^{+}\left((m-1) T_{1}\right)\right) \|_{L^{\infty}(G)} \\
& \leq \sigma_{0}\left\|u\left(T_{2} ;(m-2) T_{1}, u^{+}\left((m-2) T_{1}\right)\right)\right\|_{L^{\infty}(G)} \\
& \leq \cdots \leq \sigma_{0}^{m-1}\left\|u\left(T_{2} ; 0, u^{+}(0)\right)\right\|_{L^{\infty}(G)} .
\end{aligned}
$$

Further, by Corollary 6.9

$$
\left\|u\left(T_{2} ; 0, u^{+}(0)\right)\right\|_{L^{\infty}(G)} \leq \tilde{C}_{10}\left\|u^{+}(0)\right\|_{L^{\infty}(G)} \frac{\left\|\varphi_{G}\left(T_{2}\right)\right\|_{L^{\infty}(G)}}{\left\|\varphi_{G}(0)\right\|_{L^{\infty}(G)}} .
$$

Combining the above inequalities and using (7.38), we obtain

$$
\left\|u^{+}\left(T_{2}\right)\right\|_{L^{\infty}(G)}<\frac{1}{2}\left\|u^{+}(0)\right\|_{L^{\infty}(G)} \frac{\left\|\varphi_{G}\left(T_{2}\right)\right\|_{L^{\infty}(G)}}{\left\|\varphi_{G}(0)\right\|_{L^{\infty}(G)}} .
$$

The same estimate holds if we replace " $u$ " " by " $u$ " ". We therefore have

$$
\begin{aligned}
\left\|u\left(T_{2}\right)\right\|_{L^{\infty}(G)} & \leq \max \left\{\left\|u^{+}\left(T_{2}\right)\right\|_{L^{\infty}(G)},\left\|u^{-}\left(T_{2}\right)\right\|_{L^{\infty}(G)}\right\} \\
& <\frac{1}{2} \max \left\{\left\|u^{+}(0)\right\|_{L^{\infty}(G)},\left\|u^{-}(0)\right\|_{L^{\infty}(G)}\right\} \frac{\left\|\varphi_{G}\left(T_{2}\right)\right\|_{L^{\infty}(G)}}{\left\|\varphi_{G}(0)\right\|_{L^{\infty}(G)}} \\
& =\frac{1}{2}\left\|u_{0}\right\|_{L^{\infty}(G)} \frac{\left\|\varphi_{G}\left(T_{2}\right)\right\|_{L^{\infty}(G)}}{\left\|\varphi_{G}(0)\right\|_{L^{\infty}(G)}} .
\end{aligned}
$$

It remains to prove (7.37) under the assumption (ii) above. In that case, using (ii), Corollary 6.9 and Corollary [5.3, we derive

$$
\begin{aligned}
\frac{\left\|u\left(T_{2}\right)\right\|_{L^{\infty}(G)}}{\left\|u_{0}\right\|_{L^{\infty}(G)}} & =\frac{\left\|u\left(T_{2}\right)\right\|_{L^{\infty}(G)}}{\left\|u\left(t_{0}+\tau_{0}\right)\right\|_{L^{\infty}(G)}} \frac{\left\|u\left(t_{0}+\tau_{0}\right)\right\|_{L^{\infty}(G)}}{\left\|u\left(t_{0}\right)\right\|_{L^{\infty}(G)}} \frac{\left\|u\left(t_{0}\right)\right\|_{L^{\infty}(G)}}{\left\|u_{0}\right\|_{L^{\infty}(G)}} \\
& \leq \tilde{C}_{10} \frac{\left\|\varphi_{G}\left(T_{2}\right)\right\|_{L^{\infty}(G)}}{\left\|\varphi_{G}\left(t_{0}+\tau_{0}\right)\right\|_{L^{\infty}(G)}} \frac{\left(\tilde{C}_{2}\right)^{5}}{2\left(\tilde{C}_{10}\right)^{2}} \tilde{C}_{10} \frac{\left\|\varphi_{G}\left(t_{0}\right)\right\|_{L^{\infty}(G)}}{\left\|\varphi_{G}(0)\right\|_{L^{\infty}(G)}} \\
& \leq \frac{\left(\tilde{C}_{2}\right)^{5}}{2} \frac{\left\|\varphi_{G}\left(t_{0}\right)\right\|_{L^{\infty}(G)}}{\left\|\varphi_{G}\left(t_{0}+\tau_{0}\right)\right\|_{L^{\infty}(G)}} \frac{\left\|\varphi_{G}\left(T_{2}\right)\right\|_{L^{\infty}(G)}}{\left\|\varphi_{G}(0)\right\|_{L^{\infty}(G)}} \\
& \leq \frac{1}{2} \frac{\left\|\varphi_{G}\left(T_{2}\right)\right\|_{L^{\infty}(G)}}{\left\|\varphi_{G}(0)\right\|_{L^{\infty}(G)}},
\end{aligned}
$$

finishing the proof of Lemma 7.3 .

Lemma 7.3 implies the following theorem.

Theorem 7.4. Let $G$ be a smooth domain such that $D_{2} \subset \subset G \subseteq \Omega$ and $\operatorname{dist}\left(\bar{D}_{2}, \partial G\right) \geq d>0$. Let $u$ be a solution of (3.1), (3.2) on $G \times\left[s_{0}, \infty\right)$ with $u\left(\cdot, s_{0}\right) \in C_{0}(\bar{G})$ and such that $u(\cdot, t)$ vanishes somewhere in $G$ for all $t>s_{0}$. There exist positive constants $C, \gamma$, depending only on $\operatorname{inrad}(\Omega), \operatorname{diam}(\Omega), D_{0}, d_{0}$, $D_{1}, d_{1}, D_{2}, d$ and the structure of $L$, such that the following holds:

$$
\frac{\|u(t)\|_{L^{\infty}(G)}}{\left\|\varphi_{G}(t)\right\|_{L^{\infty}(G)}} \leq C e^{-\gamma(t-s)} \frac{\|u(s)\|_{L^{\infty}(G)}}{\left\|\varphi_{G}(s)\right\|_{L^{\infty}(G)}} \quad\left(t \geq s \geq s_{0}\right) .
$$

Proof. Let $T_{2}$ be as in Lemma 7.3 and $s \geq s_{0}$. First, by the maximum principle, we have $\|u(t)\|_{L^{\infty}(G)} \leq e^{\beta_{0} T_{2}}\|u(s)\|_{L^{\infty}(G)}$ for any $t \in\left[s, s+T_{2}\right]$. Further, by Corollary 5.3 with $D=D_{0}$ we also have $\left\|\varphi_{G}(t)\right\|_{L^{\infty}(G)} \geq\left(\tilde{C}_{2}\right)^{T_{2}+1}\left\|\varphi_{G}(s)\right\|_{L^{\infty}(G)}$ for all 
$t \in\left[s, s+T_{2}\right]$, where $\tilde{C}_{2}$ depends only on $\operatorname{inrad}(\Omega), D_{0}, d_{0}$ and the structure of $L$. These inequalities give

$$
\frac{\|u(t)\|_{L^{\infty}(G)}}{\left\|\varphi_{G}(t)\right\|_{L^{\infty}(G)}} \leq \frac{e^{\beta_{0} T_{2}}}{\left(\tilde{C}_{2}\right)^{T_{2}+1}} \frac{\|u(s)\|_{L^{\infty}(G)}}{\left\|\varphi_{G}(s)\right\|_{L^{\infty}(G)}} \quad\left(t \in\left[s, s+T_{2}\right], s \geq s_{0}\right) .
$$

For any $t \geq s \geq s_{0}$ there are a unique integer $j$ and $a \in\left[0, T_{2}\right)$ such that $t=$ $s+j T_{2}+a$. The above estimate and Lemma 7.3 then imply

$$
\frac{\|u(t)\|_{L^{\infty}(G)}}{\left\|\varphi_{G}(t)\right\|_{L^{\infty}(G)}} \leq \frac{e^{\beta_{0} T_{2}}}{\left(\tilde{C}_{2}\right)^{T_{2}+1}} \frac{\left\|u\left(s+j T_{2}\right)\right\|_{L^{\infty}(G)}}{\left\|\varphi_{G}\left(s+j T_{2}\right)\right\|_{L^{\infty}(G)}} \leq \frac{e^{\beta_{0} T_{2}}}{2^{j}\left(\tilde{C}_{2}\right)^{T_{2}+1}} \frac{\|u(s)\|_{L^{\infty}(G)}}{\left\|\varphi_{G}(s)\right\|_{L^{\infty}(G)}},
$$

which can be written as in (7.42) for appropriate $C, \gamma>0$ depending only on $\operatorname{inrad}(\Omega), \operatorname{diam}(\Omega), D_{0}, d_{0}, D_{1}, d_{1}, D_{2}, d$ and the structure of $L$.

\section{Proof of Theorem 2.2 and Corollary 2.4}

In this section we fix $\varphi_{\Omega}$, a positive entire solution of (1.1) given by Theorem 2.1, We defer the proof of uniqueness of $\varphi_{\Omega}$ to the next section and we keep this solution fixed throughout this section. Also, in the following text, $D_{0}, d_{0}, D_{1}, d_{1}$, and $D_{2}$ are as chosen in the previous sections. Let us first prove the following lemma.

Lemma 8.1. Let $G, d$ be as in Lemma 7.1, For each $u_{0} \in C_{0}(\bar{G})$ and $s \in \mathbb{R}$ there exist a unique $q_{0} \in \mathbb{R}$ and $\zeta_{0} \in C_{0}(\bar{G})$ such that (with $\varphi_{G}$ as in Theorem 3.8)

$$
u_{0}(x)=q_{0} \frac{\varphi_{G}(x, s)}{\left\|\varphi_{G}(s)\right\|_{L^{\infty}(G)}}+\zeta_{0}(x) \quad(x \in G),
$$

and such that the solution $u\left(\cdot, t ; s, \zeta_{0}\right)$ of (3.1), (3.2) on $G \times[s, \infty)$ vanishes somewhere in $G$ for all $t>s$. Moreover, we have the following estimate:

$$
\left|q_{0}\right| \leq C_{13}|| u_{0} \|_{L^{\infty}(G)}
$$

and the constant $C_{13}>0$ depends only on $\operatorname{inrad}(\Omega), \operatorname{diam}(\Omega), D_{0}, d_{0}, D_{1}, d_{1}, D_{2}$, $d$ and the structure of $L$.

Proof. The existence and uniqueness of the decomposition (8.1) follows from [17, Theorem 2.6]. We thus only need to prove the estimate (8.2). Define the solution

$$
\tilde{u}(x, t)=u\left(x, t ; s, u_{0}\right)+C \frac{\varphi_{G}(x, t)}{\left\|\varphi_{G}(s)\right\|_{L^{\infty}(G)}} \quad(x \in G, t \geq s) .
$$

Set $\kappa=C_{1}\left(D_{2}, d\right)$, where $C_{1}\left(D_{2}, d\right)$ is as in Lemma 5.2. With this $\kappa$ we apply Lemma 6.6 with $t_{0}=s$ to $v(\cdot, t)=C \varphi_{G}(\cdot, t) /\left\|\varphi_{G}(s)\right\|_{L^{\infty}(G)}$ and $u(\cdot, t)=$ $u\left(\cdot, t ; s, u_{0}\right)$. We obtain that if $C>0$ exceeds $C_{13}\left\|u_{0}\right\|_{L^{\infty}(G)}$, where $C_{13}=$ $\left(C_{1}\left(D_{2}, d\right) C_{9}\left(D_{2}, d\right)\right)^{-1}$ depends only on $\operatorname{inrad}(\Omega), \operatorname{diam}(\Omega), D_{0}, d_{0}, D_{1}, d_{1}, D_{2}$, $d$ and the structure of $L$, then $\tilde{u}(x, t)>0$ for all $x \in \bar{D}_{2}$ and all $t>s+1$. Hence, by Proposition 6.11 $\tilde{u}(x, t)>0$ for all $(x, t) \in G \times\left[t_{G}(\tilde{u}), \infty\right)$, and the properties of the decomposition (8.1) then imply that $\tilde{u}(x, t) \not \equiv u\left(x, t ; s, \zeta_{0}\right)$. Thus, necessarily $-q_{0}<C_{13}\left\|u_{0}\right\|_{L^{\infty}(G)}$. Replacing "+" in (8.3) by "-", we also get $q_{0}<C_{13}\left\|u_{0}\right\|_{L^{\infty}(G)}$, finishing the proof.

Define the set

$$
\tilde{X}^{2}(s)=\left\{u_{0} \in X(s): \frac{\left\|u\left(\cdot, t ; s, u_{0}\right)\right\|_{L^{\infty}(\Omega)}}{\left\|\varphi_{\Omega}(t)\right\|_{L^{\infty}(\Omega)}} e^{\gamma(t-s)} \text { is bounded for } t \geq s\right\},
$$


where $X(s)$ is as in (4.24) and $\gamma>0$ is as in Theorem 7.4 with $d=d_{2} / 2$, where

$$
d_{2}=\operatorname{dist}\left(\bar{D}_{2}, \partial \Omega\right)
$$

From (8.4) and the linear dependence of $u\left(\cdot, t ; s, u_{0}\right)$ on $u_{0}$ it follows that $\tilde{X}^{2}(s)$ is a subspace of $X(s)$. We will now show that

$$
X(s)=\operatorname{span}\left\{\varphi_{\Omega}(\cdot, s)\right\} \oplus \tilde{X}^{2}(s) \quad(s \in \mathbb{R}) .
$$

Let $u_{0} \in X(s)$. Let $\Omega_{j} \subset \subset \Omega_{j+1} \subset \subset \Omega$ be a sequence of smooth subdomains such that $\bigcup_{j=1}^{\infty} \Omega_{j}=\Omega$ and assume without loss of generality that $\operatorname{dist}\left(\bar{D}_{2}, \partial \Omega_{j}\right) \geq d_{2} / 2$ for all $j \geq 1$. Furthermore, let $\xi_{j} u_{0}$ be the approximating initial conditions as defined in Proposition 4.13, For each $j \geq 1$ we can write by Lemma 8.1 .

$$
\xi_{j}(x) u_{0}(x)=q_{j} \frac{\varphi_{\Omega_{j}}(x, s)}{\left\|\varphi_{\Omega_{j}}(s)\right\|_{L^{\infty}\left(\Omega_{j}\right)}}+\zeta_{j 0}(x) \quad\left(x \in \Omega_{j}\right)
$$

and $\left|q_{j}\right| \leq C_{13}|| \xi_{j} u_{0}\left\|_{L^{\infty}\left(\Omega_{j}\right)} \leq C_{13}|| u_{0}\right\|_{L^{\infty}(\Omega)}$ and $u\left(\cdot, t ; s, \zeta_{j 0}\right)$ is a sign-changing solution on $\Omega_{j} \times(s, \infty)$. We also note that our assumption on $\Omega_{j}$ implies that $C_{13}$ depends only on $\operatorname{inrad}(\Omega), \operatorname{diam}(\Omega), D_{0}, d_{0}, D_{1}, d_{1}, D_{2}, d_{2}$ and the structure of $L$. By the boundedness of $\left|q_{j}\right|$ we can choose a subsequence (keeping the same notation) such that $q_{j} \rightarrow q_{0}$ for some $q_{0} \in \mathbb{R}$. The proof of Theorem 5.5 shows that (a subsequence of) the functions $\varphi_{\Omega_{j}}(\cdot, s) /\left\|\varphi_{\Omega_{j}}(s)\right\|_{L^{\infty}\left(\Omega_{j}\right)}$ converge locally uniformly in $\Omega$ to $\varphi_{\Omega}(\cdot, s) /\left\|\varphi_{\Omega}(s)\right\|_{L^{\infty}(\Omega)}$. Therefore

$$
\xi_{j} u_{0}-q_{j} \frac{\varphi_{\Omega_{j}}(s)}{\left\|\varphi_{\Omega_{j}}(s)\right\|_{L^{\infty}\left(\Omega_{j}\right)}}=\zeta_{j 0} \rightarrow u_{0}-q_{0} \frac{\varphi_{\Omega}(s)}{\left\|\varphi_{\Omega}(s)\right\|_{L^{\infty}(\Omega)}}=: \zeta_{0}
$$

locally uniformly in $\Omega$. Since $u\left(\cdot, t ; s, \zeta_{j 0}\right)$ is a sign-changing solution on $\Omega_{j} \times(s, \infty)$ and $\operatorname{dist}\left(\bar{D}_{2}, \partial \Omega_{j}\right) \geq d_{2} / 2$, we may apply Theorem 7.4 with $G=\Omega_{j}$ and $d=d_{2} / 2$ to get

$$
\frac{\left\|u\left(\cdot, t ; s, \zeta_{j 0}\right)\right\|_{L^{\infty}\left(\Omega_{j}\right)}}{\left\|\varphi_{\Omega_{j}}(t)\right\|_{L^{\infty}\left(\Omega_{j}\right)}} \leq C e^{-\gamma(t-s)} \frac{\left\|\zeta_{j 0}\right\|_{L^{\infty}\left(\Omega_{j}\right)}}{\left\|\varphi_{\Omega_{j}}(s)\right\|_{L^{\infty}\left(\Omega_{j}\right)}} \quad(t \geq s, j \geq 1)
$$

where $C$ and $\gamma>0$ depend only on $\operatorname{inrad}(\Omega), \operatorname{diam}(\Omega), D_{0}, d_{0}, D_{1}, d_{1}, D_{2}, d_{2}$ and the structure of $L$. In particular, these constants do not depend on $j$, and we note that the $\gamma$ here is the same as in (8.4). Letting $j \rightarrow \infty$ and using Proposition 4.13 (note that $\zeta_{0} \in X(s)$ ) and (8.8), we obtain

$$
\frac{\left\|u\left(\cdot, t ; s, \zeta_{0}\right)\right\|_{L^{\infty}(\Omega)}}{\left\|\varphi_{\Omega}(t)\right\|_{L^{\infty}(\Omega)}} \leq C e^{-\gamma(t-s)} \frac{\left\|\zeta_{0}\right\|_{L^{\infty}(\Omega)}}{\left\|\varphi_{\Omega}(s)\right\|_{L^{\infty}(\Omega)}} \quad(t \geq s) .
$$

This in particular gives that $\zeta_{0} \in \tilde{X}^{2}(s)$. Therefore any $u_{0} \in X(s)$ can be written as $u_{0}=q_{0} \varphi_{\Omega}(s) /\left\|\varphi_{\Omega}(s)\right\|_{L^{\infty}(\Omega)}+\zeta_{0}$ for some $q_{0} \in \mathbb{R}$ and $\zeta_{0} \in \tilde{X}^{2}(s)$ and the solution $u\left(\cdot, t ; s, \zeta_{0}\right)$ satisfies (8.9). The uniqueness of this decomposition clearly follows from the definition of $\tilde{X}^{2}(s)$, and the proof of (8.6) is finished.

We add that the above arguments also prove that (8.9) holds for all $\zeta_{0} \in \tilde{X}^{2}(s)$. This implies that $\tilde{X}^{2}(s)$ is closed in $X(s)$. Indeed, consider a sequence $u_{j} \in \tilde{X}^{2}(s)$ approaching some $u_{0} \in X(s)$. It follows from (8.9) that the expression in (8.4) with $u_{0}$ replaced by $u_{j}$ is bounded by a constant independent of $j$. Taking the limit we obtain that the expression with $u_{0}$ is bounded; hence $u_{0} \in \tilde{X}^{2}(s)$. 
We would like to prove now that $\tilde{X}^{2}(s)=X^{2}(s), s \in \mathbb{R}$, where $X^{2}(s)$ is defined as in Theorem 2.2, i.e.,

$$
X^{2}(s)=\left\{u_{0} \in X(s): \text { for all } T>s \text { the function } u\left(\cdot, t ; s, u_{0}\right)\right.
$$

Let us first prove the following implication:

$$
u_{0} \notin X^{2}(s) \Rightarrow u_{0} \notin \tilde{X}^{2}(s) .
$$

Let $u_{0} \in X(s)$ be such that $u_{0} \notin X^{2}(s)$, i.e., without loss of generality,

$$
u\left(x, t ; s, u_{0}\right)>0 \quad\left(x \in \bar{D}_{2}, t \geq t_{0}\right),
$$

where $t_{0}$ is some sufficiently large number. Since both sets $X^{2}(s)$ and $\tilde{X}^{2}(s)$ are clearly invariant with respect to (1.1) $\left(u_{0} \in X^{2}(s)\right.$ implies $u\left(\cdot, t ; s, u_{0}\right) \in X^{2}(t)$ for all $t \geq s$ ), we may assume $t_{0}=s$ and for simplicity of notation we will take $s=0$. Let $u_{j}$ be the approximating solutions considered in Proposition 4.13 and let $\Omega_{j}$, $j \geq 1$ be as specified above. The local uniform convergence of these solutions to $u$ along with (8.12) (with $t_{0}=s=0$ ) implies that for each $T>1$ there exists an integer $j_{0}(T)$ such that for all $j \geq j_{0}(T)$ we have

$$
u_{j}(x, t)>0 \quad\left(x \in \bar{D}_{2}, t \in[0, T+1]\right),
$$

and with $x_{\Omega}$ and $c_{\Omega}$ as in (5.1) we have

$$
u_{j}\left(x_{\Omega}, t\right)>\frac{u\left(x_{\Omega}, t\right)}{2} \geq e^{-\left(\log c_{\Omega}\right)(t-1)} \frac{u\left(x_{\Omega}, 1\right)}{2} \quad(t \in[2, T+1]),
$$

for all $j \geq j_{0}(T)$ where we have used the Harnack inequality in the second inequality above. Moreover, by Lemma 3.5 (with " $U=\Omega_{j} \backslash \bar{D}_{2}$ ") we have for all $j \geq j_{0}(T)$,

$$
\begin{aligned}
\left\|u_{j}^{-}(t)\right\|_{L^{\infty}\left(\Omega_{j} \backslash \bar{D}_{2}\right)} & \leq 2 e^{-k t}\left\|u_{j}^{-}(0)\right\|_{L^{\infty}\left(\Omega_{j} \backslash \bar{D}_{2}\right)} \\
& \leq 2 e^{-k t}\left\|u_{0}\right\|_{L^{\infty}(\Omega)} \quad(t \in[0, T+1]),
\end{aligned}
$$

where $k$ is as in (7.3), and the second inequality above follows from the definition of $u_{j}$. Since $k>\log c_{\Omega}$ and $\operatorname{dist}\left(\bar{D}_{2}, \partial \Omega_{j}\right) \geq d_{2} / 2$, the inequalities (8.14), (8.15) imply that we can choose $T>1$ so large (depending on $u$ ) that for any $j \geq j_{0}(T)$ all the assumptions in Theorem 3.6 are satisfied with $\rho=\operatorname{inrad}(\Omega) / 4, d=d_{2} / 4$, $\theta=1 / 8, D=D_{2}, G=\Omega_{j}, v=u_{j}$ and $\tau=T$. Therefore, for all $j \geq j_{0}(T)$, we have

$$
u_{j}(x, t)>0 \quad\left(x \in \bar{D}_{2}, t \in[T, \infty)\right) .
$$

Note that this inequality (and (8.13) ) implies that (8.15) holds for all $t \geq 0$. Observe now that by the same arguments as those leading to (8.16), possibly increasing $T$ if necessary, one proves that (8.16) remains valid if we replace $u_{j}(\cdot, t)$ by $u_{j}(\cdot, t)-$ $\alpha \varphi_{\Omega_{j}}(\cdot, t) /\left\|\varphi_{\Omega_{j}}(T)\right\|_{L^{\infty}\left(\Omega_{j}\right)}$ for some small $\alpha>0$ depending on $u$ but not on $j$ as long as $j \geq j_{0}(T)$. Therefore

$$
u_{j}(x, t) \geq \alpha \frac{\varphi_{\Omega_{j}}(x, t)}{\left\|\varphi_{\Omega_{j}}(T)\right\|_{L^{\infty}\left(\Omega_{j}\right)}} \quad\left(x \in \bar{D}_{2}, t \geq T, j \geq j_{0}(T)\right) .
$$

Using Lemma 5.2 (with $D=D_{2}$ and $G=\Omega_{j}$ ) and taking the limit $j \rightarrow \infty$ in (8.17), we obtain for some $C>0$,

$$
\frac{\|u(t)\|_{L^{\infty}(\Omega)}}{\left\|\varphi_{\Omega}(t)\right\|_{L^{\infty}(\Omega)}} \geq \alpha \frac{C}{\left\|\varphi_{\Omega}(T)\right\|_{L^{\infty}(\Omega)}} \quad(t \geq T)
$$

which implies that $u_{0} \notin \tilde{X}^{2}(s)$. Therefore (8.11) holds and hence $\tilde{X}^{2}(s) \subseteq X^{2}(s)$. 
Let us now prove the opposite inclusion. Let $u_{0} \in X(s)$. By (8.6) we can write $u_{0}=q_{0} \varphi_{\Omega}(s)+\zeta_{0}$ for some $q_{0} \in \mathbb{R}$ and $\zeta_{0} \in \tilde{X}^{2}(s)$. Suppose $q_{0}>0$, for example. The estimate (8.9), which has been shown to hold for all $\zeta_{0} \in \tilde{X}^{2}(s)$, and Theorem 5.5 with $D=D_{2}$ imply that $u\left(x, t ; s, u_{0}\right)>0$ for all $x \in \bar{D}_{2}$ and $t \geq t_{0}>s$, where $t_{0}$ is a sufficiently large number. Therefore $u_{0} \notin X^{2}(s)$, showing that for $u_{0} \in X^{2}(s)$ we must have $q_{0}=0$ and thus $X^{2}(s) \subseteq \tilde{X}^{2}(s)$. Therefore $X^{2}(s)=\tilde{X}^{2}(s)$ for all $s \in \mathbb{R}$. This equality and (8.6) and (8.9) yield both (i) and (ii) in Theorem 2.2.

Proof of Corollary 2.4. For any $s \in \mathbb{R}$ define the set

$$
\begin{gathered}
Z(s)=\left\{u_{0} \in X(s): \text { for all } T>s \text { the function } u\left(\cdot, t ; s, u_{0}\right)\right. \\
\text { has a zero in } \bar{D} \text { for some } t \geq T\} .
\end{gathered}
$$

Since $D_{2} \subseteq D$ we obviously have $X^{2}(s) \subseteq Z(s)(s \in \mathbb{R})$. Let $u_{0} \in X(s)$. By (2.2) we can write $u_{0}=q_{0} \varphi_{\Omega}(s)+\zeta_{0}$ for some $q_{0} \in \mathbb{R}$ and $\zeta_{0} \in X^{2}(s)$. Suppose $q_{0}>0$, for example. The estimate (2.3), applied to " $u_{0}=\zeta_{0}$ ", and Theorem [5.5] imply that $u\left(x, t ; s, u_{0}\right)>0$ for all $x \in \bar{D}$ and $t \geq t_{0}>s$, where $t_{0}$ is a sufficiently large number. Therefore $u_{0} \notin Z(s)$, showing that for $u_{0} \in Z(s)$ we must have $q_{0}=0$ and thus $Z(s) \subseteq X^{2}(s)$. Hence $Z(s)=X^{2}(s)$ for all $s \in \mathbb{R}$.

\section{Proof of Proposition 2.5}

It is sufficient to show that any positive entire solution $\varphi_{1}$ of (1.1), which satisfies (2.5), is a constant multiple of $\varphi_{\Omega}$, where $\varphi_{\Omega}$ is as chosen at the beginning of the previous section. Indeed, the solution $\varphi_{\Omega}$ satisfies (2.5) for some $c>0$. This is a simple consequence of Theorem [5.5. Corollary 6.5 (see also Corollary 6.10) and the maximum principle (Corollary 4.11). From now on we may therefore assume that (2.5) holds for both $\varphi_{\Omega}$ and $\varphi_{1}$ with the same $c>0$. With $x_{\Omega}$ as in (5.1), we shall also assume

$$
\varphi_{1}\left(x_{\Omega}, 0\right)=\varphi_{\Omega}\left(x_{\Omega}, 0\right)=1,
$$

and prove that necessarily $\varphi_{1}=\varphi_{\Omega}$. Note that this will also prove the uniqueness statement at the beginning of Theorem 2.2 .

For any smooth subdomain $D$ in $\Omega$ such that $D_{2} \subset \subset D \subset \subset \Omega$ and $\operatorname{dist}\left(\bar{D}_{2}, \partial D\right) \geq$ $d>0$ we claim the following is true. There exists a constant $C(D)>0$, depending only on $D, \Omega, d$ and the structure of $L$, such that

$$
C(D) \varphi_{\Omega}(x, t)-\varphi_{1}(x, t)>0 \quad((x, t) \in \bar{D} \times(-\infty,-2]) .
$$

Indeed, suppose that for some $x_{0} \in \bar{D}, t_{0} \leq-2$ and $C_{0}>0$ we had $\varphi_{1}\left(x_{0}, t_{0}\right)$ $\geq C_{0} \varphi_{\Omega}\left(x_{0}, t_{0}\right)$. It would then follow from Theorem 5.5 that $\varphi_{1}\left(x_{0}, t_{0}\right) \geq$ $C_{0} C_{4}(D)\left\|\varphi_{\Omega}\left(t_{0}\right)\right\|_{L^{\infty}(\Omega)}$. Proposition 4.13 implies that Lemma 6.6 holds with $G=\Omega$ if we replace $C_{9}(D, d)$ by, say, $C_{9}(D, d / 2) / 2$. Using Lemma 6.6 with

$$
G=\Omega, \kappa=\frac{\left\|\varphi_{1}(t)\right\|_{L^{\infty}(D)}}{\left\|\varphi_{1}(t)\right\|_{L^{\infty}(\Omega)}}, v=\varphi_{1}, u=\varphi_{\Omega},
$$

we get that if $C_{0} C_{4}(D) \geq 2 C_{9}^{-1}(D, d / 2)$, then $\varphi_{1}(x, t)>\varphi_{\Omega}(x, t)$ for all $x \in \bar{D}$ and $t \in\left[t_{0}+1, \infty\right)$; in particular we would get $\varphi_{1}\left(x_{\Omega}, 0\right)>\varphi_{\Omega}\left(x_{\Omega}, 0\right)$, contradicting (9.1). Thus $C_{0}<2 C_{4}^{-1}(D) C_{9}^{-1}(D, d / 2)$, which shows that (9.2) holds with $C(D)=$ $2 C_{4}^{-1}(D) C_{9}^{-1}(D, d / 2)$. 
We next claim that if $|\Omega \backslash \bar{D}|$ is sufficiently small, then the inequality (9.2) extends to $\Omega \times \mathbb{R}$. To prove this, we define the function $v_{D}(x, t)=C(D) \varphi_{\Omega}(x, t)-\varphi_{1}(x, t)$ for $(x, t) \in \Omega \times \mathbb{R}$. Then $v_{D}$ is a solution of (1.1) on $\Omega \times \mathbb{R}$ and it satisfies

$$
v_{D}>0 \text { on } \bar{D} \times(-\infty,-2] \text { and }\left|v_{D}(x, t)\right| \leq(C(D)+1) c e^{c|t|} \text { on } \Omega \times \mathbb{R},
$$

where $c>0$ is as in (2.5). The first inequality in (9.4) follows from (9.2) and the second from the discussion in the first paragraph of this section. Let $k>c$ and let $\delta>0$ be the corresponding number given by Lemma 3.5. Suppose, in addition to $D_{2} \subset \subset D \subset \subset \Omega$, that $D$ is smooth and such that $|\Omega \backslash \bar{D}|<\delta$. Again using approximation by solutions on smooth subdomains via Proposition 4.13 , in view of the first inequality in (9.4) and Lemma 3.5, one obtains the following estimate:

$$
\left\|v_{D}^{-}(t)\right\|_{L^{\infty}(\Omega)} \leq 2 e^{-k(t-\tau)}\left\|v_{D}^{-}(\tau)\right\|_{L^{\infty}(\Omega)} \quad(\tau \leq t \leq-2) .
$$

It follows from (9.4), (9.5) and the fact that $k>c$ that necessarily $v_{D}^{-}(x, t)=0$ for all $(x, t) \in \Omega \times(-\infty,-2]$. Hence, by Proposition 4.9, $v_{D} \geq 0$ on $\Omega \times \mathbb{R}$, which means $\varphi_{1} \leq C(D) \varphi_{\Omega}$ on $\Omega \times \mathbb{R}$.

By (i) in Theorem 2.2 for each $s \in \mathbb{R}$ there exist a unique $q(s) \in \mathbb{R}$ and $\zeta_{0}(\cdot, s) \in$ $X^{2}(s)$ such that

$$
0 \leq C(D) \varphi_{\Omega}(x, s)-\varphi_{1}(x, s)=q(s) \varphi_{\Omega}(x, s)+\zeta_{0}(x, s) \quad(x \in \Omega) .
$$

Proposition 4.13 implies that Lemma 8.1 holds with $G=\Omega$. Therefore

$$
|q(s)| \leq C_{13} \frac{\left\|C(D) \varphi_{\Omega}(s)-\varphi_{1}(s)\right\|_{L^{\infty}(\Omega)}}{\left\|\varphi_{\Omega}(s)\right\|_{L^{\infty}(\Omega)}} \leq C_{13} C(D),
$$

where $C_{13}$ is as in Lemma 8.1 with $d=\operatorname{dist}\left(\bar{D}_{2}, \partial D\right) / 2$.

Now fix $t, s \in \mathbb{R}$ with $t \geq s$. Applying (2.3) to $u(\cdot, t)=(C(D)-q(s)) \varphi_{\Omega}(\cdot, t)-$ $\varphi_{1}(\cdot, t)$ and using (9.6) and (9.7), we get the following estimate:

$$
\begin{aligned}
\frac{\|u(t)\|_{L^{\infty}(\Omega)}}{\left\|\varphi_{\Omega}(t)\right\|_{L^{\infty}(\Omega)}} & \leq C e^{-\gamma(t-s)} \frac{\left\|\zeta_{0}(s)\right\|_{L^{\infty}(\Omega)}}{\left\|\varphi_{\Omega}(s)\right\|_{L^{\infty}(\Omega)}} \\
& \leq C e^{-\gamma(t-s)} \frac{\left\|C(D)\left(1+C_{13}\right) \varphi_{\Omega}(s)\right\|_{L^{\infty}(\Omega)}}{\left\|\varphi_{\Omega}(s)\right\|_{L^{\infty}(\Omega)}} \\
& =C C(D)\left(1+C_{13}\right) e^{-\gamma(t-s)} .
\end{aligned}
$$

This inequality holds for any $t \geq s, t, s \in \mathbb{R}$. By (9.7) we can choose a sequence $s_{j} \rightarrow-\infty$ such that $q\left(s_{j}\right) \rightarrow q$ for some $q \in \mathbb{R}$. Taking $s=s_{j} \rightarrow-\infty$ in the above estimate and recalling our definition of $u$, we obtain $\varphi_{1}(x, t)=(C(D)-q) \varphi_{\Omega}(x, t)$ for all $(x, t) \in \Omega \times \mathbb{R}$. Finally, by (9.1) we have $C(D)-q=1$, finishing the proof.

\section{Proof of Corollary 2.6}

Let us first prove (i). We will use the fact that $u$ is a solution of $u_{t}-L u=0$ on $\Omega \times \mathbb{R}$ if and only if $v(x, t)=u(x, t) e^{\lambda t}$ is a solution of $u_{t}-L u=\lambda u$ on $\Omega \times \mathbb{R}$. Let $\varphi_{\Omega}$ be as given by Theorem 2.2. For future use, we note that by Theorem 2.1. Corollary 6.5 (see also Corollary 6.10), and Corollary 4.11 we have

$$
c e^{-\log c_{\Omega}(t-s)} \leq \frac{\left\|\varphi_{\Omega}(t)\right\|_{L^{\infty}(\Omega)}}{\left\|\varphi_{\Omega}(s)\right\|_{L^{\infty}(\Omega)}} \leq e^{\beta_{0}(t-s)} \quad(t \geq s),
$$

where $c_{\Omega}$ is as in (5.1) and $c>0$ depends only on $\Omega$ and the structure of $L$. 
By the $T$-periodicity of the coefficients of $L$, the function $u(x, t)=\varphi_{\Omega}(x, t+T)$ is also a positive entire solution of (1.1). Proposition 2.5 (and (10.1)) imply that $\varphi_{\Omega}(x, t+T)=q \varphi_{\Omega}(x, t)$ for some $q>0$ and all $(x, t) \in \Omega \times \mathbb{R}$. Set $\lambda_{1}=-(\ln q) / T$. Then $\varphi_{1}(x, t)=\varphi_{\Omega}(x, t) e^{\lambda_{1} t}$ is a positive solution of $u_{t}-L u=\lambda_{1} u$ on $\Omega \times \mathbb{R}$ and it is easy to check that $\varphi_{1}(x, t)=\varphi_{1}(x, t+T)$ for all $(x, t) \in \Omega \times \mathbb{R}$. This proves the existence of $\lambda_{1}$. Moreover, by Proposition 2.5 the eigenfunction $\varphi_{1}$ is unique and, by its definition and Theorem [2.1] it satisfies (2.1). The proof of (i) is complete.

It follows from the construction of $\lambda_{1}$ above and from (10.1) that $-\beta_{0} \leq \lambda_{1} \leq$ $\log c_{\Omega}$, proving (ii).

A straightforward modification of [17, Proof of Corollary 2.5] yields (iii), and we omit the details.

\section{REFERENCES}

[1] J.M. Arrieta. Elliptic equations, principal eigenvalue and dependence on the domain. Comm. Partial Differential Equations , 21:971-991, 1996. MR1391529(97h:35037)

[2] H. Berestycki and L. Nirenberg. On the method of moving planes and the sliding method. Bol. Soc. Brasil. Mat. (N.S.), 22:1-37, 1991. MR.1159383 (93a:35048)

[3] H. Berestycki, L. Nirenberg, and S. R. S. Varadhan. The principal eigenvalue and maximum principle for second-order elliptic operators in general domains. Comm. Pure Appl. Math., 47:47-92, 1994. MR1258192 (95h:35053)

[4] I. Birindelli. Hopf's lemma and anti-maximum principle in general domains. J. Differential Equations, 119(2):450-472, 1995. MR1340547 (969:35047)

[5] S.-N. Chow, K. Lu, and J. Mallet-Paret. Floquet theory for parabolic differential equations. J. Differential Equations, 109:147-200, 1994. MR1272403 (95c:35116)

[6] S.-N. Chow, K. Lu, and J. Mallet-Paret. Floquet bundles for scalar parabolic equations. Arch. Rational Mech. Anal., 129:245-304, 1995. MR1328478 (96c:35070)

[7] D. Daners. Existence and perturbation of principal eigenvalues for a periodic-parabolic problem. Electron. J. Diff. Eqns., Conf. 05:51-67, 2000. MR.1799044 (2001j:35125)

[8] D.-E. Edmunds, L.-A. Peletier. Removable singularities of solutions of quasi-linear parabolic equations. J. London Math. Soc., (2) 2:273-283, 1970. MR0259365 (41:4003)

[9] T. Godoy and U. Kaufmann. On principal eigenvalues for periodic parabolic problems with optimal condition on the weight function. J. Math. Anal. Appl., 262(1):208-220, 2001. MR:1857223 (2002g:35162)

[10] M. Gruber. Harnack inequalities for solutions of general second order parabolic equations and estimates of their Hölder constants. Math. Z., 185(1):23-43, 1984. MR724044 (86b:35089)

[11] P. Hess. Periodic-parabolic boundary value problems and positivity. Longman Scientific \& Technical, Harlow, 1991. MR1100011 (92h:35001)

[12] P. Hess and P. Poláčik. Boundedness of prime periods of stable cycles and convergence to fixed points in discrete monotone dynamical systems. SIAM J. Math. Anal., 24:1312-1330, 1993. MR.1234018 (94i:47087)

[13] J. Húska. Exponential separation and principal Floquet bundles for linear parabolic equations on general bounded domains: The divergence case. Indiana Univ. Math. J., 55(3):1015-1044, 2006. MR2244596

[14] J. Húska. Harnack inequality and exponential separation for oblique derivative problems on Lipschitz domains. J. Differential Equations, 226:541-557, 2006. MR 2237690

[15] J. Húska and P. Poláčik. The principal Floquet bundle and exponential separation for linear parabolic equations. J. Dynam. Differential Equations, 24:1312-1330, 2004. MR2105779 (2006e:35147)

[16] J. Húska, P. Poláčik and M.V. Safonov. Principal eigenvalues, spectral gaps and exponential separation between positive and sign-changing solutions of parabolic equations. Proceedings of the 5th International Conference on Dynamical Systems and Differential Equations, Pomona 2004, Disc. Cont. Dynamical Systems, Supplement 2005, pp. 427-435. MR2192700 (2006m:35131) 
[17] J. Húska, P. Poláčik, and M. Safonov. Harnack inequalities, exponential separation, and perturbations of principal Floquet bundles for linear parabolic equations. Ann. Inst. H. Poincaré Anal. Non Linéaire, 24:711-739, 2007. MR2348049

[18] V. Hutson, W. Shen, and G. T. Vickers. Estimates for the principal spectrum point for certain time-dependent parabolic operators. Proc. Amer. Math. Soc., 129(6):1669-1679 (electronic), 2001. MR1814096 (2001m:35243)

[19] N.V. Krylov and M.V. Safonov. A property of the solutions of parabolic equations with measurable coefficients. Izv. Akad. Nauk SSSR Ser. Mat., 44(1):161-175, 1980. MR563790 (83c:35059)

[20] N.V. Krylov. Nonlinear Elliptic and Parabolic Equations of the Second Order. Mathematics and its Applications, Reidel, Dordrecht, 1987. MR0901759 (88d:35005)

[21] G.M. Lieberman. Second order parabolic differential equations. World Scientific Publishing Co. Inc., River Edge, NJ, 1996. MR.1465184 (98k:35003)

[22] J. Mierczyński. Flows on order bundles, unpublished.

[23] J. Mierczyński. p-arcs in strongly monotone discrete-time dynamical systems. Differential Integral Equations, 7:1473-1494, 1994. MR1269666 (95c:58121)

[24] J. Mierczyński. Globally positive solutions of linear PDEs of second order with Robin boundary conditions. J. Math. Anal. Appl., 209:47-59, 1997. MR1444510(98c:35071)

[25] J. Mierczyński. Globally positive solutions of linear parabolic partial differential equations of second order with Dirichlet boundary conditions. J. Math. Anal. Appl., 226:326-347, 1998. MR1650236 (99m:35096)

[26] J. Mierczyński. The principal spectrum for linear nonautonomous parabolic pdes of second order: Basic properties. J. Differential Equations, 168:453-476, 2000. MR.1808456 (2001m:35147)

[27] J. Mierczyński and W. Shen. Exponential separation and principal Lyapunov exponent/spectrum for random/nonautonomous parabolic equations. J. Differential Equations, 191:175-205, 2003. MR.1973287 (2004h:35232)

[28] K. Miller. Barriers on Cones for Uniformly Elliptic Operators, Ann. Mat. Pura Appl., 76:93106, 1967. MR0221087 (36:4139)

[29] M. Nishio. The uniqueness of positive solutions of parabolic equations of divergence form on an unbounded domain. Nagoya Math. J., 130:111-121, 1993. MR1223732 (94f:35058)

[30] P. Poláčik. Estimates of solutions and asymptotic symmetry for parabolic equations on bounded domains. Arch. Rational Mech. Anal., 183:59-91, 2007. MR2259340

[31] P. Poláčik. On uniqueness of positive entire solutions and other properties of linear parabolic equations. Discrete Contin. Dynamical Systems, 12:13-26, 2005. MR2121246 (2005k:35170)

[32] P. Poláčik and I. Tereščák. Convergence to cycles as a typical asymptotic behavior in smooth strongly monotone discrete-time dynamical systems. Arch. Rational Mech. Anal., 116:339360, 1992. MR:1132766 (93b:58088)

[33] P. Poláčik and I. Tereščák. Exponential separation and invariant bundles for maps in ordered Banach spaces with applications to parabolic equations. J. Dynamics Differential Equations, 5:279-303, 1993. Erratum: 6(1):245-246 1994. MR1223450 (94d:47064) MR1262730

[34] W. Shen and Y. Yi. Almost automorphic and almost periodic dynamics in skew-product semiflows. Mem. Amer. Math. Soc., 647:93 p., 1998. MR.1445493 (99d:34088)

[35] I. Tereščák. Dynamics of $C^{1}$ smooth strongly monotone discrete-time dynamical systems. preprint.

[36] I. Tereščák. Dynamical systems with discrete Lyapunov functionals. Ph.D. thesis, Comenius University, 1994.

School of Mathematics, University of Minnesota, Minneapolis, Minnesota 55455

E-mail address: huska@math.umn.edu 